Highlights

- Multi-directional NewWave focused wave groups are simulated numerically and the results compared to experimental measurements

- Harmonic decomposition is used to reveal the harmonic structure of the multidirectional wave groups, including free error waves that impact extreme run-up

- Using wave generation corrected to second order minimises the impact of the free error waves, allowing more accurate predictions of extreme run-up of spread seas.

- Wavelets provide an interesting way of studying the nonlinear interactions of wave group components as they propagate up the beach

- The shoaling and run-up of long waves in a spread sea is examined 


\title{
Multi-directional focused wave group interactions with a plane beach
}

\author{
Frances M. Judge $^{* 1}$, Alison C. Hunt-Raby ${ }^{2}$, Jana Orszaghova ${ }^{3}$, Paul H. Taylor ${ }^{3}$, and \\ Alistair G. L. Borthwick ${ }^{4}$ \\ ${ }^{1}$ MaREI Centre, University College Cork, Ireland \\ ${ }^{2}$ School of Engineering, University of Plymouth, UK \\ ${ }^{3}$ Faculty of Engineering and Mathematical Sciences, University of Western Australia, Australia \\ ${ }^{4}$ Institute for Energy Systems, School of Engineering, The University of Edinburgh, UK
}

\begin{abstract}
Numerical simulations and laboratory measurements are presented of multi-directional focused wave groups interacting with a plane beach. The numerical model is a two-dimensional-horizontal (2DH) hybrid flow solver, governed by a Boussinesq equation set with enhanced dispersion characteristics pre-breaking, and the nonlinear shallow water equations post-breaking. Waves are introduced into the model via an in-built multi-element piston wavemaker, allowing for complete reproduction of laboratory experiments. A wetting and drying algorithm models shoreline movement in both cross-shore and longshore directions. Predicted free surface motions of the multi-directional focused wave groups are in good agreement with wave gauge data from laboratory experiments previously carried out at the UK Coastal Research Facility (UKCRF) using a linear paddle wave generator. Both phase decomposition into Stokes-like harmonic components and wavelets provide insight into nonlinear interactions as the wave groups propagate up the beach. Free second-order error waves in a multi-directional wave group are smaller than for the corresponding uni-directional case, and spread laterally around the incoming wave group. Of the free error waves generated by linear paddle signals, only the low-frequency second-order error wave affects extreme run-up on the beach. By applying a second-order correction to the paddle signals used to generate a multidirectional wave group, it is shown that, whereas the long error wave causes a significant increase in the maximum run-up, the impact is not as severe as for the uni-directional analogue. Shoaling and run-up of the bound long waves in a spread sea are studied. Examination of the transverse structure of these subharmonic components reveals that sideways spreading in the inner surf zone contributes to reduced run-up in directionally spread groups.
\end{abstract}

\section{Introduction}

Coastal engineers are required to analyse complicated hydrodynamic processes in the nearshore zone in order to design effective coastal protection schemes. Numerous strategies exist to model the propagation of waves over coastal bathymetry as it shallows, and the associated run-up at beaches and overtopping of sea defences. Run-up and overtopping have been investigated through field observations and laboratory tests by Holman (1986), and van der Meer and Stam (1992). However, such measurement campaigns are both expensive and labour-intensive. With coastal practitioners in mind, efforts have been made to develop empirical formulae for run-up and overtopping, such as proposed by Hunt (1959) for breaking wave run-up on smooth uniform slopes in relatively deep

\footnotetext{
*Corresponding author. Email address: frances.judge@ucc.ie
} 
water. Overtopping is often calculated using mean discharge rates as opposed to individual overtopping events (see for example the CLASH database (De Rouck et al., 2009)). The most recent EurOtop manual (van der Meer et al., 2016) recognises the limitations of using mean-discharge to describe overtopping, and emphasises the importance of the statistical distribution of overtopping volumes. Although empirical formulae for run-up and overtopping lead to useful design parameters, such calculations do not provide information on detailed spatial and temporal variations. In practice, the quantification of large individual run-up and overtopping events is important for determining sea defence failure modes, and assessing hazards posed to pedestrians and vehicles (Bruce et al., 2002). Over the past twenty years, phase-resolving numerical models based on the nonlinear shallow water equations, the Navier-Stokes equations, and the Boussinesq equations have been increasingly used to model wave propagation, run-up, and overtopping (see e.g. Schäffer et al. (1993), Hubbard and Dodd (2002), and Higuera et al. (2014)).

The nonlinear shallow water equations (NSWEs) underpin many numerical models applied to coastal engineering and large-scale flooding problems. Dodd (1998) applied the NSWEs to random wave run-up and overtopping; however the non-dispersive nature and underlying hydrostatic assumption of the NSWEs mean that they are only applicable to domains containing very shallow water where the velocity can be assumed to be nearly horizontal. Examples of two-dimensional numerical models based on the NSWEs are those of Fraccarollo and Toro (1995), Rogers et al. (2001) and Liang and Borthwick (2009). Dispersive nonlinear shallow water equations (Antuono et al., 2009) aim to merge the main advantages of the NSWEs with Boussinesq-type equations to give an equation set with both nonlinear and dispersive properties that can be solved at low computational cost.

Eulerian mesh-based computational fluid dynamics (CFD) models based on continuity and the Navier-Stokes equations offer an accurate means of predicting wave transformation, breaking, runup, and overtopping. However, such models incur very high computational cost. In practice, simplifications are incorporated to reduce the computational burden, such as the Reynolds-Averaged Navier-Stokes equations (RANS) (e.g. Lin and Liu, 1998) and Large-Eddy Simulation (LES) (see Zhou et al., 2014). An alternative Lagrangian, particle-based approach is provided by Smoothed Particle Hydrodynamics (SPH) (see e.g. Dalrymple and Rogers, 2006). In SPH methods, the fluid mass is discretised into constituent particles with assigned physical quantities; such models are inherently capable of dealing with complex wave-shore-structure interactions.

Boussinesq-type models are widely used in coastal engineering due to their ability to represent adequately the main physical processes at the shore while remaining relatively computationally efficient compared to CFD solvers. Comprehensive reviews of Boussinesq-type models are given by Madsen and Schäffer (1999), Kirby (2003), and Brocchini (2013). Hybrid Boussinesq-shallow flow models implement Boussinesq equations pre-breaking and shallow water equations post-breaking. This approach is adopted by Tonelli and Petti (2009), Tonelli and Petti (2012), Shi et al. (2012), Orszaghova et al. (2012), McCabe et al. (2013) and Judge et al. (2018). Tatlock et al. (2018) implement the roller approach in a finite difference-finite volume scheme to solve the Boussinesq equations in the surf zone, removing the need to switch to the NSWEs.

Focused wave groups offer an efficient means for coastal engineers to model large wave events at the coast, and thus estimate extreme run-up and overtopping. In particular, the NewWave methodology (see e.g. Jonathan and Taylor, 1997; Taylor and Williams, 2004; Tromans et al., 1991) ensures that the focused wave group corresponds to the average shape of the largest wave event in a Gaussian sea state, which is imperative for accurate calculations of run-up and overtopping. Whittaker et al. (2016) confirmed the validity of NewWave in relatively shallow coastal waters.

This paper aims to use a hybrid Boussinesq-shallow flow model (Judge et al., 2018, following Orszaghova 2011 and Orszaghova et al. 2012) to replicate selected multi-directional focused wave group experiments undertaken previously at the UKCRF (Hunt, 2003) based on the NewWave methodology. Stokes-like decomposition and wavelet analysis are used to investigate the harmonic structure of the multi-directional wave groups, and provide insight into the component interactions as the groups travel up the beach. A comparison is included between first-order and second-order wave generation in order to examine the significance of the long error wave from linear generation in the context of multi-directional wave groups, in particular the impact on run-up. The evolution 
of the long waves associated with a multi-directional wave group as they propagate up the beach is also examined.

\section{Background and methodology}

\subsection{First and second order wave generation in a laboratory}

Piston paddles are commonly used to generate waves in shallow and intermediate depth laboratory flumes and basins. The paddles move horizontally according to a displacement time-series calculated using wavemaker theory to produce waves with desired amplitude and frequency. First-order wavemaker theory is derived directly from linear wave theory, with additional lateral boundary conditions accounting for the moving wavemaker. For an overview, see Dean and Dalrymple (1991) or Hughes (1993). All water waves, except in the limit of infinitesimal amplitude contain nonlinearities. When paddle displacement signals calculated from first-order theory are used to generate waves in the laboratory, spurious waves are created due to the mismatch between the generated nonlinear waves and the linear paddle signal. These higher-order free parasitic or error waves are not bound to the underlying linear components and propagate independently according to their dynamics. The largest parasitic waves are sub-harmonic and super-harmonic waves associated with second-order bound Stokes terms (for details see Hunt, 2003). When generating wave groups with a linear paddle signal, the super-harmonic error waves travel slower than the main wave group. The low-frequency sub-harmonic error wave, however, travels ahead of the wave group as a single hump and thus has an impact on the wave group transformation. Schäffer (1996) derives wavemaker theory correct to second order, for normally-propagating irregular waves, for both piston and hinged wavemakers, with the aim of suppressing the generation of second-order error waves. An overview of the application of the theory is given by Orszaghova et al. (2014). Schäffer and Steenberg (2003) extend second-order wavemaker theory to multi-directional irregular waves.

\subsection{NewWave theory}

A focused wave group comprises a number of individual sinusoidal wave components that come into phase at a single point in time and space to produce a large event. Using linear wave theory, the surface elevation at any time and any point in space of a multi-directional focused wave group, where waves approach the focus location $x_{f}$ from a range of angles, is given by

$$
\zeta(x, y, t)=\sum_{n=1}^{N} a_{n} \sum_{m=1}^{M} b_{m} \cos \left(k_{n}\left(\left(x-x_{f}\right) \cos \theta_{m}+y \sin \theta_{m}\right)-\omega_{n}\left(t-t_{f}\right)+\phi\right),
$$

where $N$ is the number of wave components, $a_{n}$ is the wave amplitude, $M$ is the number of spreading angles, $b_{m}$ is a spreading factor, $k_{n}$ is the wave number, $x_{f}$ is the focus location, $\theta_{m}$ is the angle of the $m^{\text {th }}$ component measured from the $x$-axis, $\omega_{n}$ is the angular frequency, $t_{f}$ is the focus time, $\phi$ is the phase angle of the wave group.

Herein, the spreading factor is given by a top-hat function, $b(\theta)=c$, where $c$ is a constant, in accordance with the laboratory tests where no components could be created at angles too far from normal to the front face of the paddles. The formulation meets the requirement $\sum_{m=0}^{M} b_{m}=1$.

According to NewWave theory, the surface water profile in the vicinity of an extreme wave matches the normalised autocorrelation function of the underlying sea spectrum, premultiplied by the crest height. Thus for a crest-focused wave, the NewWave time history is given by

$$
\zeta(t)=A_{\mathcal{N}} \frac{\sum_{n} S_{n}(\omega) \Delta \omega_{n} \cos \left(\omega_{n}\left(t-t_{f}\right)\right)}{\sum_{n} S_{n}(\omega) \Delta \omega_{n}},
$$

where $S_{n}(\omega)$ is the discretised underlying energy spectrum and $\Delta \omega_{n}$ is the angular frequency resolution. $A_{\mathcal{N}}$ represents the linear amplitude of the largest wave from a sea surface time series of $\mathcal{N}$ waves and is calculated from $A_{\mathcal{N}}=\sqrt{2 \sigma^{2}(\ln \mathcal{N})}$, where $\mathcal{N}$ is the number of waves and $\sigma^{2}$ is 
variance of the free surface time series. In the present work, a Pierson-Moskowitz (PM) spectrum is used, given by

$$
S(\omega)=\left(\frac{\omega_{p}}{\omega}\right)^{5} \exp \left(-\frac{5}{4}\left(\frac{\omega_{p}}{\omega}\right)^{4}\right)
$$

where $\omega$ is the angular frequency, and $\omega_{p}$ is the prescribed peak angular frequency.

Focused wave groups are created in the laboratory by offsetting individual wave components at the paddles to account for the frequency dispersion of linear water waves. At the focus location, constructive interference between the individual wave components produces a large, energetic wave group. The piston paddle signal to produce a NewWave focused wave group, to first-order, is

$$
x_{p}(y, t)=\sum_{n=1}^{N} a_{n} \sum_{m=1}^{M} \frac{b_{m}}{e_{0_{n m}}} \sin \left(k_{n}\left(\left(x-x_{f}\right) \cos \left(\theta_{m}\right)+y \sin \left(\theta_{m}\right)\right)-\omega_{n}\left(t-t_{f}\right)+\phi\right),
$$

where $e_{0_{n m}}$ is the oblique linear paddle transfer function (see Schäffer and Steenberg, 2003, for details). The wave component amplitudes necessary to generate NewWave in equation (4) are given by

$$
a_{n}=\frac{A_{\mathcal{N}} S_{n}(\omega) \Delta \omega_{n}}{\sum_{n} S_{n}(\omega) \Delta \omega_{n}}
$$

Note that a trough-focused wave group is generated by replacing $a_{n}$ with $-a_{n}$, which is equivalent to setting $\phi=\pi$ in Equation 1. To generate a spread-sea focused wave group, correct to second order, the multi-directional wavemaker theory of Schäffer and Steenberg (2003) has to be employed. Only sub-harmonic correction is implemented, because the short error waves do not affect run-up. For each pair of linear harmonic components (in Equation 1), a second order difference-frequency correction is calculated. The second-order paddle transfer function contains infinite summation terms due to taking into account the evanescent mode interactions. In practice these are evaluated up to a finite number of terms, and the asymptotic solution of Schäffer (1993b) is also used to keep the number of terms low to speed up the paddle signal calculations.

\subsection{NewWave experiments at the UKCRF}

Experiments undertaken at the UK Coastal Research Facility (UKCRF) using focused wave groups are described by Hunt (2003) and Hunt-Raby et al. (2011), where NewWave theory was implemented to generate 32 different focused wave groups with varying amplitudes and phases. The UKCRF wave basin had internal plan dimensions of $20 \mathrm{~m}$ x $36 \mathrm{~m}$ in the downwave $x$ - and lateral $y$-directions. Waves were generated by 72 independently operated piston paddles, each $0.5 \mathrm{~m}$ wide and $1.5 \mathrm{~m}$ high. The toe of a 1:20 plane beach was located $8.33 \mathrm{~m}$ from the paddles. The basin floor and plane beach had smooth, impermeable surfaces. Wave gauges positioned at $250 \mathrm{~mm}$ intervals along the basin centre-line were used to measure water surface elevation time series from $x=6.83 \mathrm{~m}(1.5 \mathrm{~m}$ offshore of the beach toe) to $x=18.33 \mathrm{~m}$ (still water shoreline location, see Figure 1). The focus point of the directionally spread wave groups was invariably located along this centre-line. The test programme encompassed uni-directional wave groups (WG1-WG16) and directionally spread wave groups (WG17-WG32) interacting with a plane beach and a sea wall. Both crest-focused and trough-focused wave groups were considered. Focus locations included: the beach toe, $3 / 4$ offshore depth (i.e. $1 / 4$ of the way up the beach), and $1 / 2$ offshore depth. Focusing of the wave groups was based on linear wave theory assuming that the waves were propagating on water of uniform depth in the absence of the beach. For the focus location at the beach toe, the presence of the beach had little effect locally, whereas focus locations further up the beach were affected by the beach slope. However, it is convenient to define focusing in terms of the above locations on the beach. It should be noted that the focus location considered in the remainder of this paper was a function of $x$ and $t$ only, because the multi-directional wave group is symmetrical about the basin centreline.

Table 1 summarises details of the selected wave groups considered in this paper. The underlying PM spectrum had a peak angular frequency $\omega_{p}=2.91 \mathrm{rad} \mathrm{s}^{-1}(0.46 \mathrm{~Hz})$. The truncated spectrum was defined by $\omega_{\min } \approx 2.07 \mathrm{rad} \mathrm{s}^{-1}(0.33 \mathrm{~Hz})$ and $\omega_{\max } \approx 6.06 \mathrm{rad} \mathrm{s}^{-1}(0.96 \mathrm{~Hz})$. This spectrum was discretised into $N=53$ components with a uniform angular frequency resolution of $\Delta \omega \approx 0.077$ 


\begin{tabular}{lllll}
\hline \multicolumn{5}{c}{ Multi-directional wave groups at the UKCRF } \\
\hline Name & $\begin{array}{l}\text { Input amplitude } \\
(\mathrm{mm})\end{array}$ & Focus location & $\begin{array}{l}\text { Spread angle } \\
(\mathrm{deg})\end{array}$ & $\begin{array}{l}\text { Phase of group } \\
(\mathrm{rad})\end{array}$ \\
\hline WG1 & 114 & beach toe & 0 & 0 \\
WG2 & 114 & $\frac{3}{4}$ offshore depth & 0 & 0 \\
WG3 & 90 & 立 offshore depth & 0 & 0 \\
WG5 & 114 & beach toe & 0 & $\pi$ \\
WG6 & 114 & $\frac{3}{4}$ offshore depth & 0 & $\pi$ \\
WG7 & 90 & $\frac{1}{2}$ offshore depth & 0 & $\pi$ \\
WG17 & 114 & beach toe & \pm 30 & 0 \\
WG18 & 114 & $\frac{3}{4}$ offshore depth & \pm 30 & 0 \\
WG19 & 90 & $\frac{1}{2}$ offshore depth & \pm 30 & 0 \\
WG21 & 114 & beach toe & \pm 30 & $\pi$ \\
WG22 & 114 & $\frac{3}{4}$ offshore depth & \pm 30 & $\pi$ \\
WG23 & 90 & $\frac{1}{2}$ offshore depth & \pm 30 & $\pi$ \\
WG25 & 114 & beach toe & \pm 10 & 0 \\
WG26 & 114 & $\frac{3}{4}$ offshore depth & \pm 10 & 0 \\
WG27 & 90 & $\frac{1}{2}$ offshore depth & \pm 10 & 0 \\
WG29 & 114 & beach toe & \pm 10 & $\pi$ \\
WG30 & 114 & $\frac{3}{4}$ offshore depth & \pm 10 & $\pi$ \\
WG31 & 90 & $\frac{1}{2}$ offshore depth & \pm 10 & $\pi$ \\
\hline
\end{tabular}

Table 1: Selected multi-directional wave group tests in the UKCRF (Hunt, 2003).

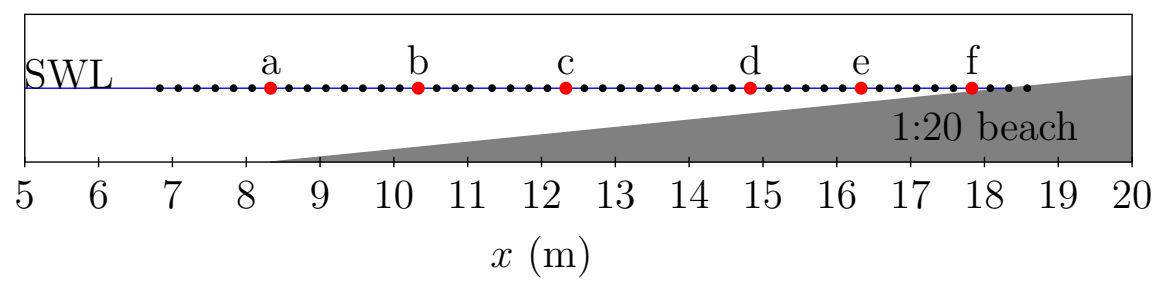

Figure 1: Gauge locations for the UKCRF run-up experiments reported by Hunt (2003). The gauge locations highlighted in red (labelled a-f) are used in Section 3.1 (Figures 3 and 4) to compare numerical and measured data. The horizontal scale denotes distance from the paddle. The offshore water depth is $0.5 \mathrm{~m}$.

$\operatorname{rad~s}^{-1}$. The top-hat spreading factor $b(\theta)=1 / M$ where the number of spread angles was $M=81$. Paddle signals were calculated according to Equation (4), with an experimentally derived transfer function used in place of the theoretical linear paddle transfer function. A detailed explanation of the paddle calibration procedure is given in Chapter 4 of Hunt (2003). The resulting experimental data have been used to verify numerical models described by Borthwick et al. (2006), Orszaghova (2011) and Orszaghova et al. (2012).

\subsection{Numerical model}

The multi-directional focused wave group experiments in the UKCRF are simulated numerically using the 2DH flow solver described by Judge et al. (2018). This numerical model is a hybrid Boussinesq - shallow flow solver that simulates the propagation of waves in two horizontal-dimensions from intermedate depth $(d / \lambda=0.5$, where $d$ is local depth and $\lambda$ is wavelength). Pre-breaking, wave propagation is calculated using the enhanced Boussinesq equation set of Madsen and Sørensen (1992). This equation set has improved dispersion characteristics; the equations are discretised using second-order central differences and solved using the conjugate gradient method with fourthorder Runge-Kutta time integration. In the breaker zone, a ramping function is applied to the 
Boussinesq dispersive terms so that they are gradually switched off, and the resulting nonlinear shallow water equations are solved using a finite volume MUSCL-Hancock scheme with an HLLC approximate Riemann solver, following Liang and Borthwick (2009). Broken waves are treated as hydraulic bores. A wetting and drying algorithm models the wet/dry front as it moves up and down the beach and also spreads laterally in the longshore direction. It does this by excluding any dry cells or volume elements from the computational process unless they are about to be flooded (i.e. the bed level of the dry cell is below that of a wet neighbouring cell to the north, south, east or west). The moving shoreline is therefore automatically tracked within the solver. Where a cell has water depth less than the critical value, then it is automatically dried out, its water redistributed into an adjacent cell, and the intercell fluxes are adjusted accordingly. For full details of the numerical implementation, refer to Liang and Borthwick (2009) or Judge (2018). The location of the switch from the Boussinesq to the shallow water equations is determined by a local calculation of the slope of the free surface in any direction. The switch point is then set to half a wavelength offshore of the point where the free surface slope exceeds a threshold value of $0.4\left(\approx 22^{\circ}\right)$, and applied evenly across the domain in the $y$-direction (parallel to the beach). The switch location is recalculated at every time step, and thus follows the breaking waves inshore.

Waves are generated in the numerical model by a line of independently moving piston paddles. This allows full replication of laboratory experiments, including the generation of oblique waves and spread seas, as well as waves that propagate normal to the shore. Wave generation is undertaken through a linear mapping, which maps the stretching and compressing domain in the region of the paddles onto a fixed domain, to circumvent re-gridding at every time step. For the initial test cases considered herein, the numerical wave paddles are driven according to the linear experimental paddle displacement signals calculated by Hunt (2003), to allow a complete reproduction of the experiments. The simulation results are presented in Sections 3.1-3.4. Additionally, for a number of selected wave groups, the paddle signals have been recalculated using second-order wavemaker theory (Schäffer and Steenberg, 2003). These are used in Section 3.4.1 to generate focused wave groups where the low-frequency error wave travelling ahead of the group has been largely removed.

Judge et al. (2018) present a series of benchmark tests to verify the numerical model, and obtain satisfactory results for nearshore circulation at sinusoidal and cusped beaches by comparing numerical predictions to the laboratory measurements of Da Silva Lima (1981) and Borthwick and Foote (2002). Numerical simulations of uni-directional focused wave interactions with a plane beach are also presented, and the results compared with Hunt's laboratory measurements (WG1 of the UKCRF tests).

\subsubsection{Model calibration}

Orszaghova (2011) previously calibrated a one-dimensional hybrid model of the UKCRF by adjusting values of the bed friction coefficient $C_{f}$, and the local free surface slope coefficient, $\Phi$, that triggers the switch from the Boussinesq to the shallow water equations, until a best fit (assessed by visual comparison of numerical predictions and gauge measurements) was obtained between the model predictions and the experimental data on focused waves. The resulting values, $C_{f}=0.008$ and $\Phi=0.4$ are also used here. Whittaker et al. (2017) perform a similar exercise in a different laboratory, and justify setting $\Phi=0.4$. The eddy viscosity coefficient, $\epsilon$, is set to zero.

\section{Results}

\subsection{Multi-directional focused wave group}

The directionally spread wave group experiments at the UKCRF reported by Hunt (2003) included waves with top-hat spreading angles of $\pm 10^{\circ}$ and $\pm 30^{\circ}$. The test case of a multi-directional focused wave group with spread angle $\pm 30^{\circ}$, linear focus amplitude $A_{f}=0.114 \mathrm{~m}$ and focus location at the beach toe, is first considered (WG17 in Hunt, 2003). Waves are generated in the numerical model using paddle displacement signals from the laboratory experiments. Here, the computational domain matches the internal basin measurements of $20 \mathrm{~m} \times 36 \mathrm{~m}$, with the grid spacing in the $x$ - 


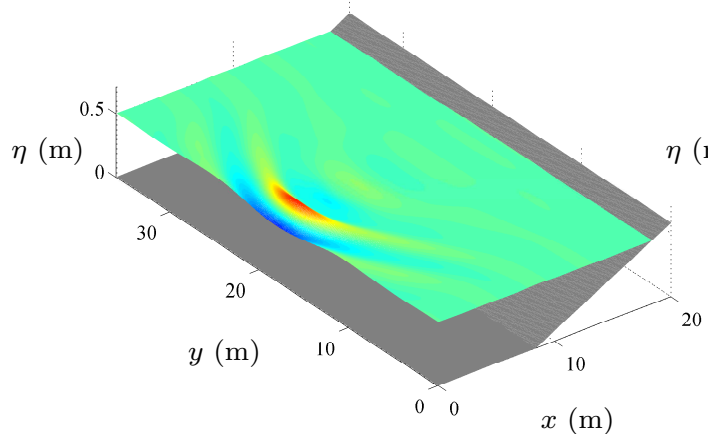

(a)

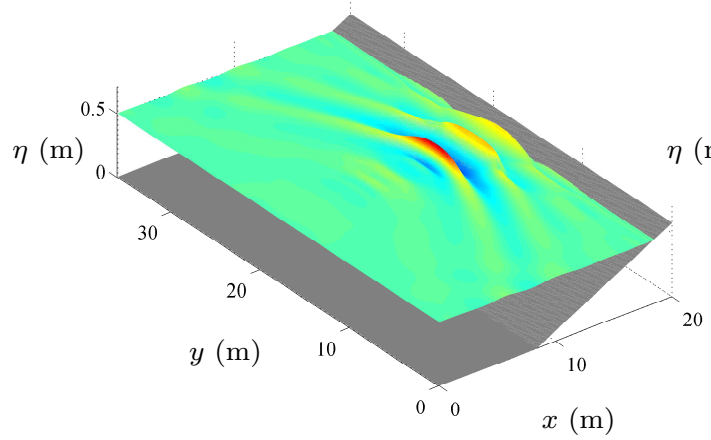

(c)

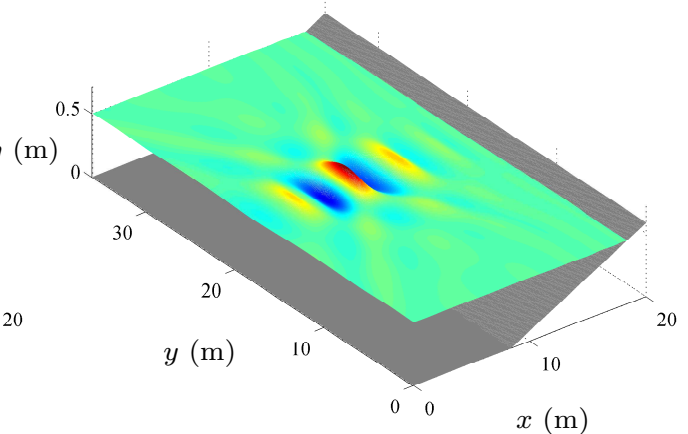

(b)

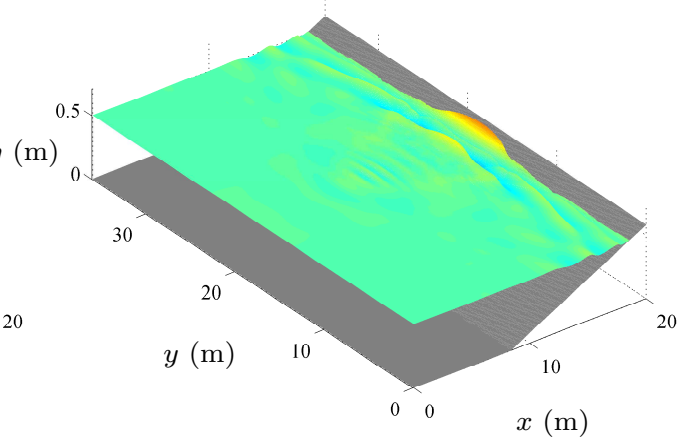

(d)

Figure 2: Numerically predicted water surface elevation distributions for $\pm 30^{\circ}$ spread multi-directional focused wave group (WG17) in the UKCRF at: (a) $t=40 \mathrm{~s}$; (b) $t=45$ $\mathrm{s} ;(\mathrm{c}) t=50 \mathrm{~s}$; and $(\mathrm{d}) t=55 \mathrm{~s}$.

direction set to $\Delta x=0.05 \mathrm{~m}$. As there are 72 paddles in the UKCRF basin, each paddle is modelled with 10 grid points in the $y$-direction, giving $\Delta y=0.0501 \mathrm{~m}$. The simulation is run for $90 \mathrm{~s}$ to match the laboratory experiment, with time step $\Delta t=0.01 \mathrm{~s}$. Linear interpolation is performed on the experimental paddle signal to produce signals with a time step matching that of the numerical model. The simulation CPU run time is approximately 17 hours 45 minutes (compiled using gfortran with -O3 optimisation on a late 2011 MacBook Pro with a $2.3 \mathrm{GHz}$ Intel Core i5 processor executing 3 threads).

In the early stages of the simulation, the amplitudes of the paddle motions are low, sending small waves across the basin. The paddle amplitudes gradually increase, producing larger amplitude wave components, with all waves coming into phase where the beach toe meets the basin centreline at $t \approx 46 \mathrm{~s}$, producing a large wave event that is now localised in both directions. Figure 2 illustrates the evolution of the free surface profile during the simulation.

Figure 3 compares the laboratory measurements and numerical predictions of the free surface elevation time series at 6 gauge locations along the UKCRF basin centre-line. The performance of the model for the multi-directional wave group is very similar to the uni-directional case presented by Judge et al. (2018): leading waves in the wave group are predicted with excellent accuracy, whereas pre-breaking, the height of the central wave crest in the group is under-predicted, and the trough following this crest is over-predicted. The transformation to a steep-fronted bore is captured very well, while a slight phasing mismatch is present in the propagation of the third and subsequent bores in very shallow water. Figure 4 shows the corresponding results obtained for the trough-focused multi-directional wave group (WG21 in Hunt, 2003). Again very satisfactory agreement is obtained between the predictions and measurements, except for slight over-predictions of the primary trough depth at focus, and the trailing trough depth as the group approaches the shoreline. 


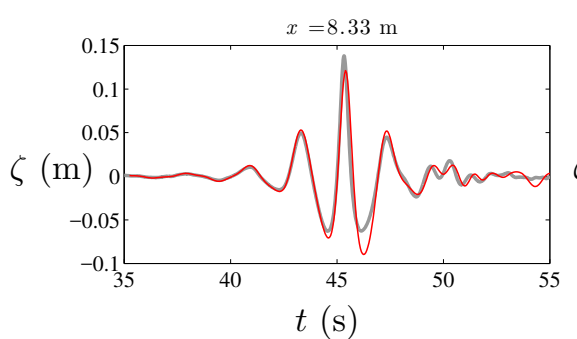

(a)

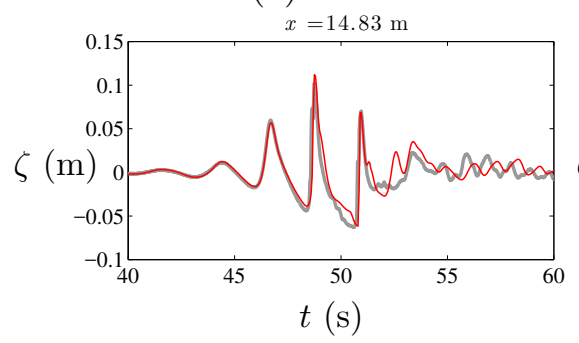

(d)

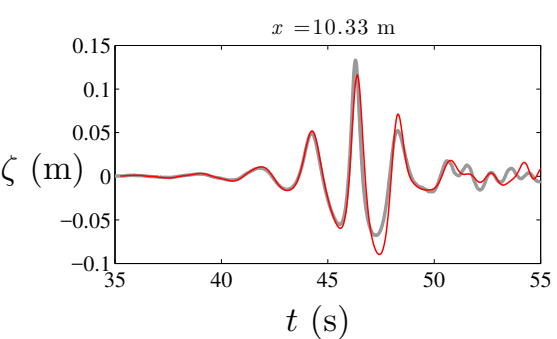

(b)

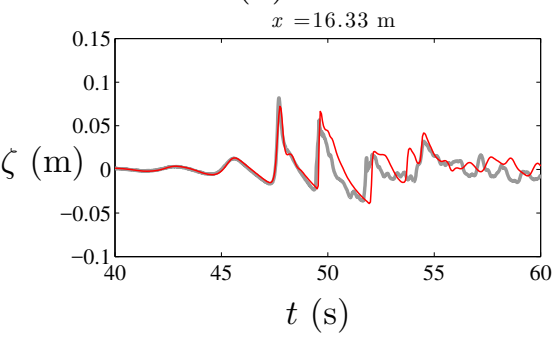

(e)

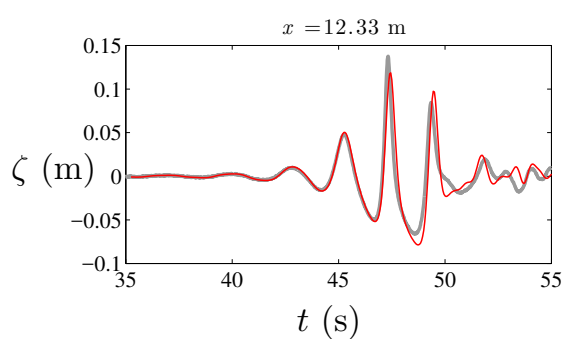

(c)

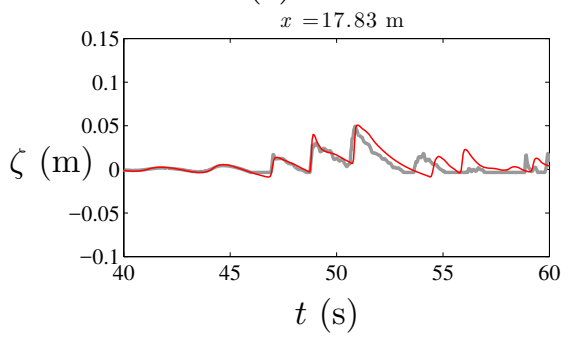

(f)

Figure 3: WG17 - Crest-focused multi-directional wave group $\left( \pm 30^{\circ}\right.$ spread $)$ in the UKCRF: comparison between measured (thick grey line) and numerically predicted (red line) free surface elevation time series at six gauge locations from $x=8.33$ to $x=17.83$ $\mathrm{m}$ along the centreline of the basin.

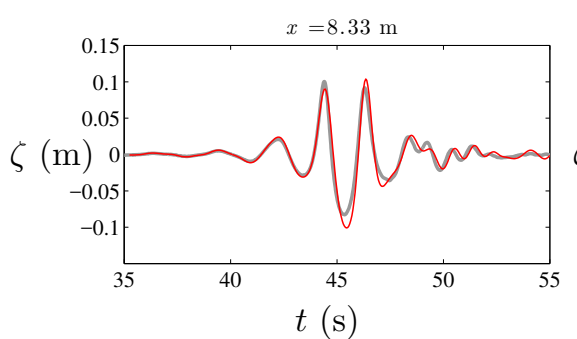

(a)

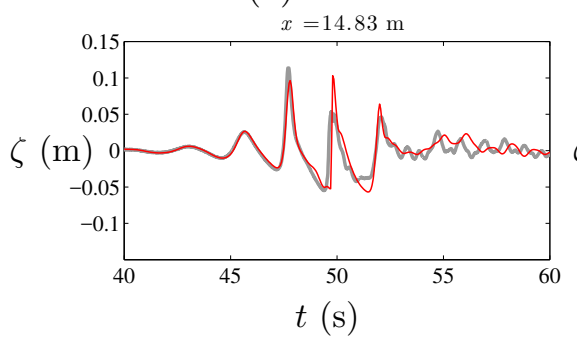

(d)

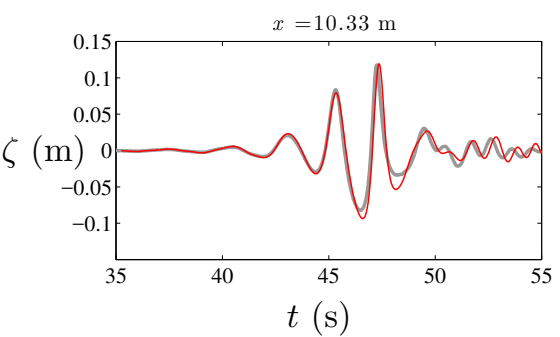

(b)

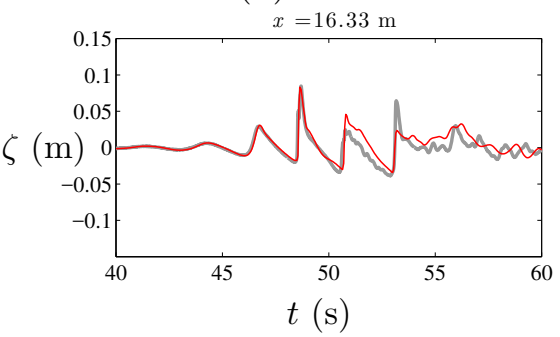

(e)

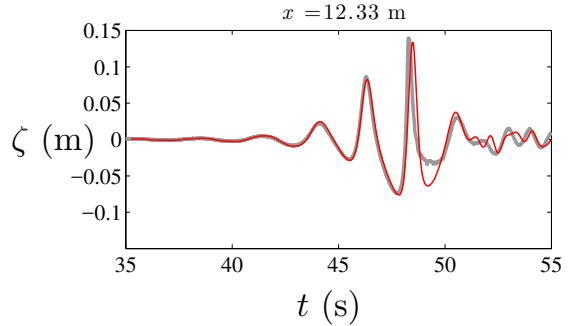

(c)

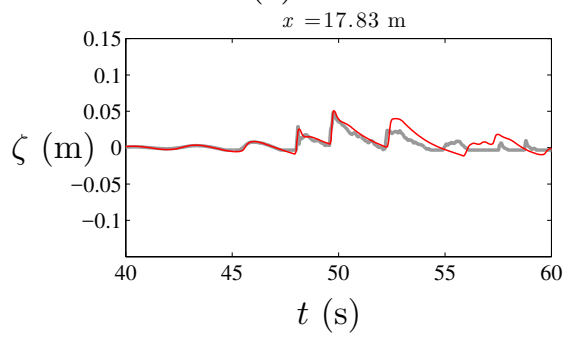

(f)

Figure 4: WG21 - Trough-focused multi-directional wave group ( $\pm 30^{\circ}$ spread $)$ in the UKCRF: comparison between measured (thick grey line) and numerically predicted (red line) free surface elevation time series at six gauge locations from $x=8.33$ to $x=17.83$ $\mathrm{m}$ along the centreline of the basin. 


\subsection{Focused wave group harmonic structure}

Waves were generated in the UKCRF experiments by supplying linear paddle signals, and therefore, contaminated by the spurious waves described in Section 2.1. Evanescent wave components generated by a piston-paddle wavemaker are non-propagating and have amplitudes that decay rapidly away from the paddle, and so are not considered further here. Hunt (2003) analysed the harmonic structure of focused wave groups travelling normal to the beach by isolating individual harmonics through judicious combination of the surface elevation data from crest- and trough-focused wave groups in the UKCRF experiments. Orszaghova et al. (2014) performed a similar exercise using data from the one-dimensional numerical model. The purpose was to find evidence of the lowfrequency free error wave manifesting itself as a hump of water travelling ahead of the main wave group that may contaminate the results, in particular those for wave run-up. In the present study, a similar exercise is performed, but for multi-directional wave groups, namely wave groups WG17 and WG21 which are crest- and trough-focused with a spread angle of $\pm 30^{\circ}$. It should be noted that the method of harmonic analysis assumes that the positions of the crests in one wave group are matched by the troughs in the inverted group, so the same locally linearised wave envelope is recovered even though the waves are themselves nonlinear and experience wave-wave interactions. Therefore, application of this method to WG17 and WG21 should work well offshore of the breaker line. Closer to the shore, crests and troughs may not be in alignment, therefore alternative methods are used in subsequent sections to examine wave behaviour in this region (see Section 3.3 on wavelet analysis and Section 3.5 on long wave propagation).

Application of a fast-Fourier-transform (FFT) to the predicted surface elevation data for WG17 and WG21 at the beach toe, leads to the amplitude spectra shown in Figure 5. Inspection of these spectra reveals that both the crest- and trough-focused time series (sub-figures (a) and (b)) have a concentration of energy at $0.46 \mathrm{~Hz}$, at the peak frequency of the linear components. Secondary peaks are visible at approximately double this peak frequency as well as in the sub-harmonic range (below $\approx 0.3 \mathrm{~Hz}$ ). Figures $5(\mathrm{c})$ and $(\mathrm{d})$ present the amplitude spectra of the addition and subtraction time series respectively at the beach toe, and can be used to identify suitable cut-off frequencies to isolate bound and parasitic higher order waves. We note that the input range of linear wave components is $0.33-0.96 \mathrm{~Hz}$. The amplitude spectrum of the addition time series which contains even-order harmonics, reduces to zero at $0.5 \mathrm{~Hz}$, which suggests that this would be a suitable cut-off point to isolate low and high frequency second-order harmonics (denoted by $2^{-}$and $2^{+}$on figure). For the subtraction time series, a cut-off frequency of $1 \mathrm{~Hz}$ is used to separate the linear, first-order terms (denoted by 1) from the higher-order harmonics (denoted by $3^{+}$).

Figure 6 presents the predicted time histories of the harmonics associated with WG17 and WG21 at the beach toe on the centreline of the basin. Figure 6 (a) is obtained by applying a low-pass filter at $1 \mathrm{~Hz}$ to the subtraction time series revealing the linear signal (denoted by 1). The second-order sum harmonics (i.e. the high frequency waves denoted by $2^{+}$) are shown in Figure 6 (b) and the second-order difference harmonics (i.e. the long waves, denoted by $2^{-}$) are shown in Figure 6 (c), obtained by applying high and low-pass filters respectively at $0.5 \mathrm{~Hz}$. In the figures, 'b' and 'e' indicate bound and error and waves respectively.

The large depression in Figure 6 (c) is the bound set-down beneath the wave group $\left(2^{-} \mathrm{b}\right)$. For wave groups in finite depth, the appearance of rather small positive peaks either side of the much larger central set-down is consistent with Fourier-based second order bound wave theory (Dalzell, 1999; Dean and Sharma, 1981). In Figure 6 (c), a very shallow hump follows the set down, and a much larger wave crest can clearly be seen travelling ahead of the main group $\left(2^{-} \mathrm{e}\right)$. This indicates the presence of a free error wave. It is likely that the appearance of low-frequency waves after $t \approx 52 \mathrm{~s}$ occurs as a result of reflections. Figure $6(\mathrm{~d})$ shows the higher-order harmonics (mainly third-order), obtained by applying a high-pass filter at $1 \mathrm{~Hz}$ to the subtraction time-series. The waves between 55 and 60 seconds are due to numerical reflections (see Figure 10a). The third and higher order bound harmonics coincide with the passage of the wave group at about $45 \mathrm{~s}$.

Figures 7 and 8 present $x-t$ visualisations of the propagation of the crest- and trough-focused wave groups WG17 and WG21 along the centreline of the basin. Both the numerical model output and the experimental gauge data are shown. Presentation of the data in this way allows an overall comparison to be made between the numerical results and the gauge data, as well as revealing fea- 


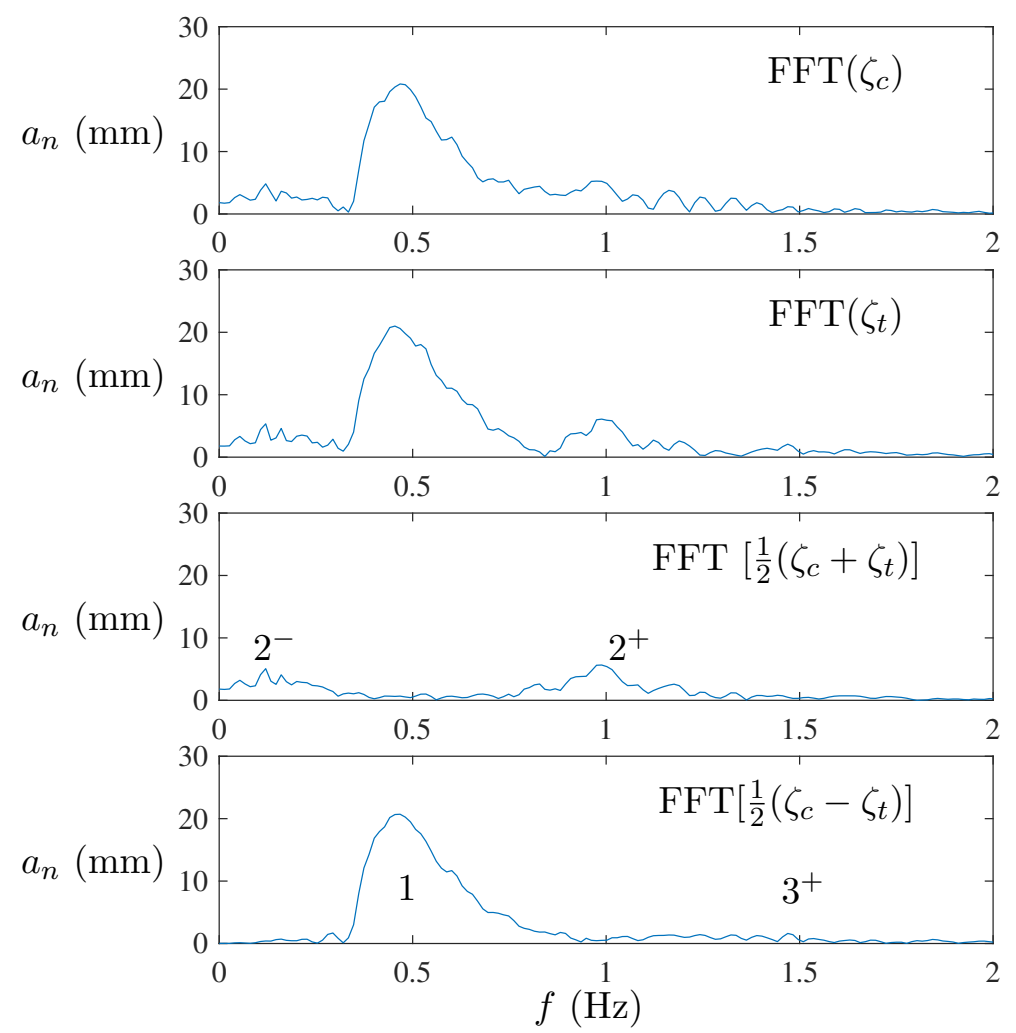

(a)

(b)

(c)

Figure 5: Predicted amplitude spectra at the beach toe for (a) crest-focused wave group time series (WG17); (b) trough-focused wave group time series (WG21); (c) addition time series; and (d) subtraction time-series

tures such as wave reflections, which are not necessarily obvious in the individual gauge comparisons shown in Figures 3 and 4 . The experimental data are restricted to gauge locations, and therefore the plots show measurements as far as $x \approx 18 \mathrm{~m}$. No such restrictions exist for the numerical plots, which show the movement of the shoreline beyond the still water level $(\mathrm{SWL}=18.33 \mathrm{~m})$. Beyond $18.33 \mathrm{~m}$, the free-surface elevations above beach level are shown, rather than above the SWL. Note that the experimental $x-t$ plots are generated from coarser resolution data, with interpolation used to facilitate comparison with the higher resolution numerical data.

In Figure 7, the central crest (shown in red) of WG17 and the troughs on either side (shown in blue) are clearly visible. The wave group focuses at $x \approx 8.33 \mathrm{~m}$, when the crests on either side of the main crest (shown in yellow/orange) are approximately the same size. The maximum run-up is caused by the central crest at $t \approx 52 \mathrm{~s}$. Dispersion is evident as the wave group propagates up the beach and becomes less well defined. Both the gauge and $x-t$ plots (Figures 3 and 7 ) reveal a slight difference between the numerical and experimental wave celerity as the waves travel up the beach, where the numerical bores travel slower than those observed in the laboratory. This may be attributed to limitations in the shallow water equations implemented in the numerical model, which predict a phase speed of $c=\sqrt{g d}$ (in which $d=h+\zeta$ where $h$ is the still water depth and $\zeta$ is the free surface elevation), where $k d<\pi / 10$. Therefore, although the NSWEs account for amplitude dispersion, the residual effects of frequency dispersion are not considered and may be the reason for the observed differences in phase speed. Breaking can be identified by a sharp drop in amplitude of the central crest at $x \approx 16 \mathrm{~m}$, and of the trailing crest at $x \approx 15 \mathrm{~m}$. In both the numerical and experimental results, there is evidence of long-wave reflections. Moreover, the numerical plot also contains some high-frequency reflections from $x \approx 12 \mathrm{~m}$. These are also present in the one-dimensional numerical results of Orszaghova (2011) and Orszaghova et al. (2014) who attribute the presence of such reflections to the switch between governing equation sets. The $x-t$ plots of the trough-focused wave group WG21 in Figure 8 exhibit similar features. Again there is evidence of low frequency reflections in both the numerical and experimental results. The maximum run-up in this case is caused by the crest leading the central trough. 


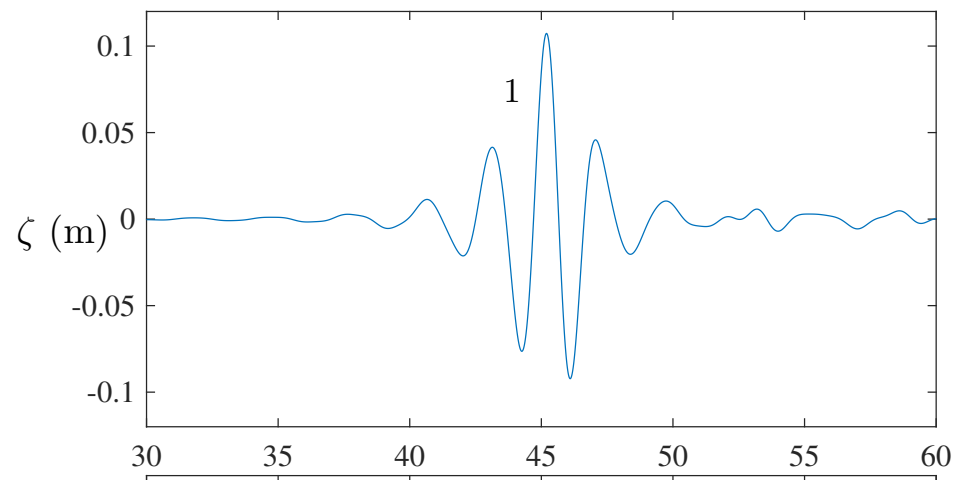

(a)

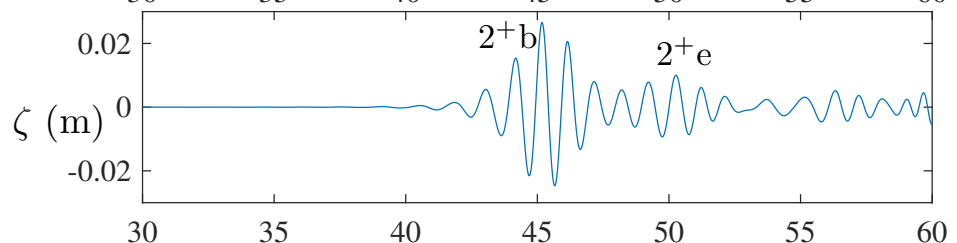

(b)

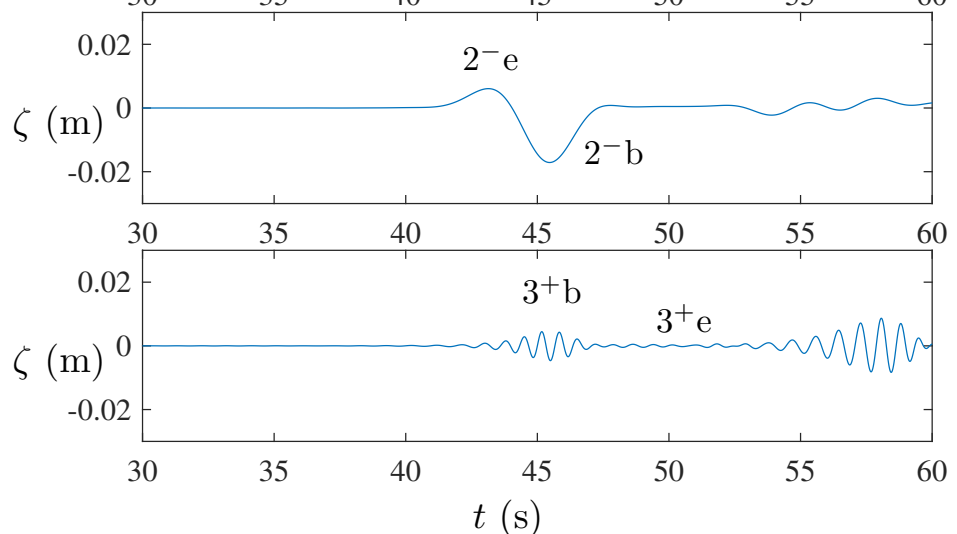

(c)

(d)

Figure 6: Predicted free-surface time histories from WG17 and WG21 at $x=8.33 \mathrm{~m}$ (beach toe): (a) linear crest and trough signal (subtraction time-series, low-pass filtered at $1 \mathrm{~Hz}$ ); (b) second-order sum harmonics (addition time-series, high-pass filtered at 0.5 $\mathrm{Hz}$ ); (c) second-order difference harmonics (long waves, i.e. addition time-series, lowpass filtered at $0.5 \mathrm{~Hz}$ ); and (d) third-order harmonics (subtraction time-series, high-pass filtered at $1 \mathrm{~Hz})$.

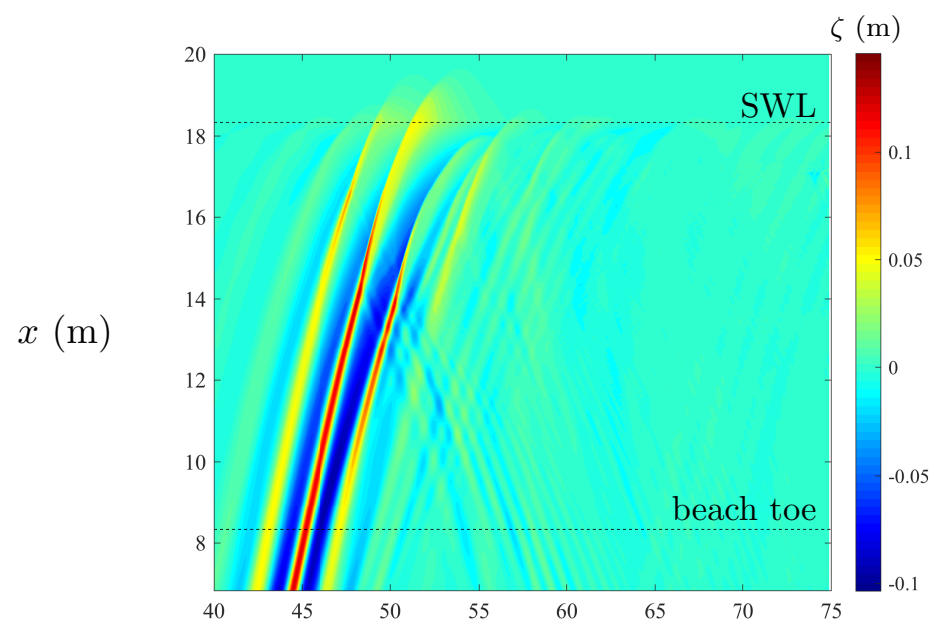

$t(\mathrm{~s})$

(a)

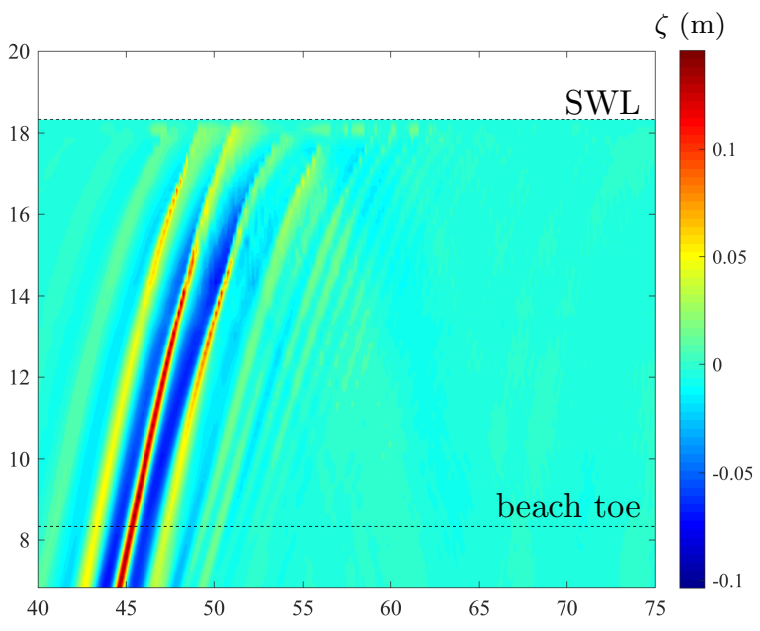

$t(\mathrm{~s})$

(b)

Figure 7: Space-time plots of NewWave propagation at a plane beach for a crest-focused wave group (WG17): (a) numerical prediction; and (b) measured free surface elevation data, along basin centreline. 


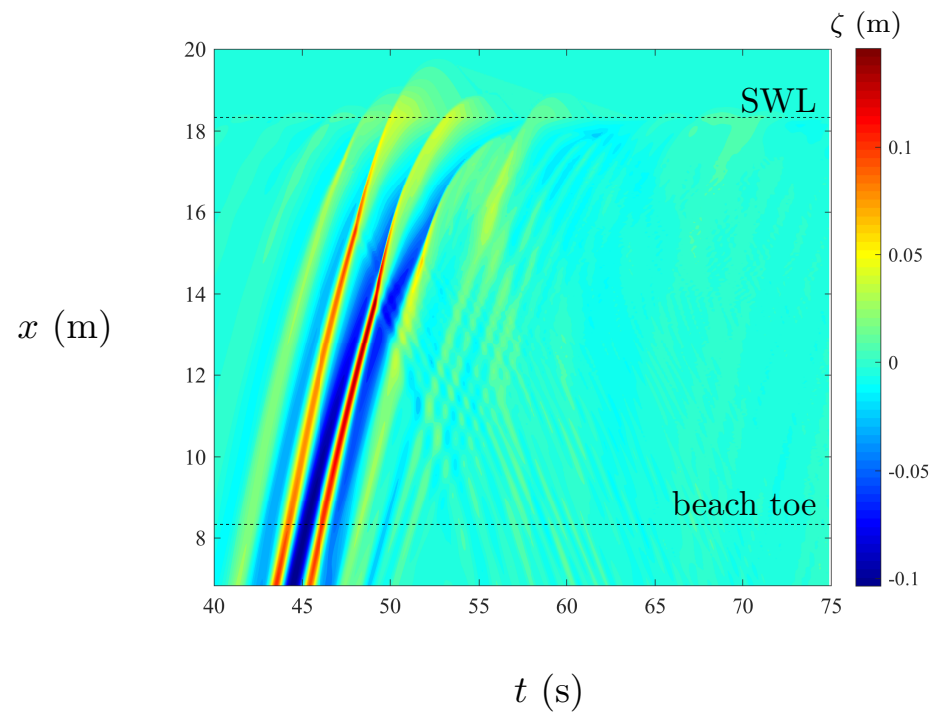

(a)

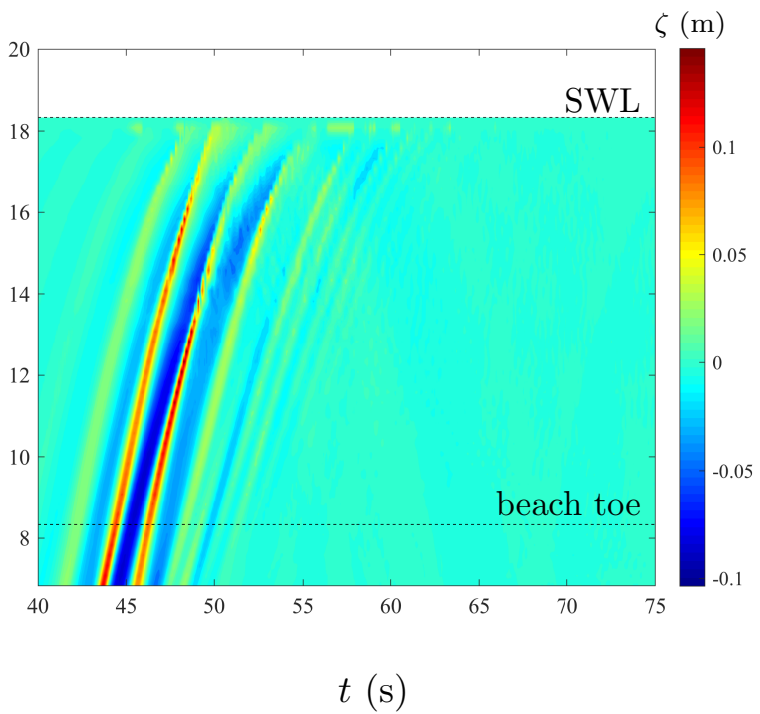

(b)

Figure 8: Space-time plots of NewWave propagation at a plane beach for a trough-focused wave group (WG21): (a) numerical prediction; and (b) measured free surface elevation data, along basin centreline.

Individual low and high frequency, odd and even harmonics are investigated further by applying the cut-off frequencies identified above in Figure 5. Figure 9 shows the linear components of wave groups WG17 and WG21. The very steep fronts of the waves that characterise the unfiltered time series have been eliminated. Unmodified by the higher-order components, the wave crests remain centrally positioned as the waves propagate into shallower water. The experimental $x-t$ plot shows no reflections, i.e. all the linear components are dissipated by the beach, whereas the numerical results contain some weak reflections at $x \approx 12 \mathrm{~m}$. Odd order sum frequency harmonics are visualised in Figure 10, revealing the third-order harmonics $\left(3^{+} \mathrm{b}\right.$ and $\left.3^{+} \mathrm{e}\right)$, and perhaps traces of the fifth $\left(5^{+} \mathrm{e}\right)$ sum harmonics. Again, reflections are evident at $x \approx 12.5 \mathrm{~m}$ in the numerical results, unlike the experimental data. These reflections are primarily due to implementation of the transition zone to the shallow water equations at wave breaking.

Figure 11 shows the $x-t$ visualisation of the even-order difference frequency harmonics, primarily second-order difference components. The blue depression in these plots represents the bound second-order wave travelling beneath the main wave group, or the 'set-down'. The yellow-orange hump preceding the bound set-down is a combination of the low frequency error wave that travels independently of the wave group, and a lower-amplitude bound wave crest. The darker blue bound wave in Figure 11a indicates that the numerical model predicts a larger set-down beneath the main wave group than observed in the laboratory. The numerical results also indicate a larger leading wave crest, which increases in amplitude slightly with respect to its experimental counterpart as the wave group approaches the shore. This is consistent with both focusing of the linear group, and reduction in water depth (the shallow water bound set-down $\approx-|A(\tilde{x})|^{2} / 4 d$, where $\tilde{x}$ is the horizontal coordinate of the group reference frame, and $d$ is the water depth; see Mei et al. (1981) or McAllister et al. (2018). At the shoreline, the amplitude of the leading wave crest increases sharply at $t \approx 50 \mathrm{~s}$, and penetrates the swash zone beyond $x=19 \mathrm{~m}$. In both the numerical and experimental plots, there is evidence of consistently raised water levels at the SWL due to low frequency wave action (indicated by the yellow horizontal line at $x=18.33 \mathrm{~m}$ ). This may be evidence of a resonant response in the swash zone triggered by the arrival of incoming waves, and is discussed further in Section 3.3 and Section 3.5. A very small trailing hump appears in both the predicted and experimental results, but is much more prominent in the former. A discrepancy grows between the predicted and measured results in the breaking zone and inshore, perhaps owing to the poorer representation of the physics by the shallow water equations and their tendency to sharpen the front of the primary hump. This has an impact on the application of harmonic decomposition, as the crests and troughs are now out of alignment and do not cancel out in the addition time 


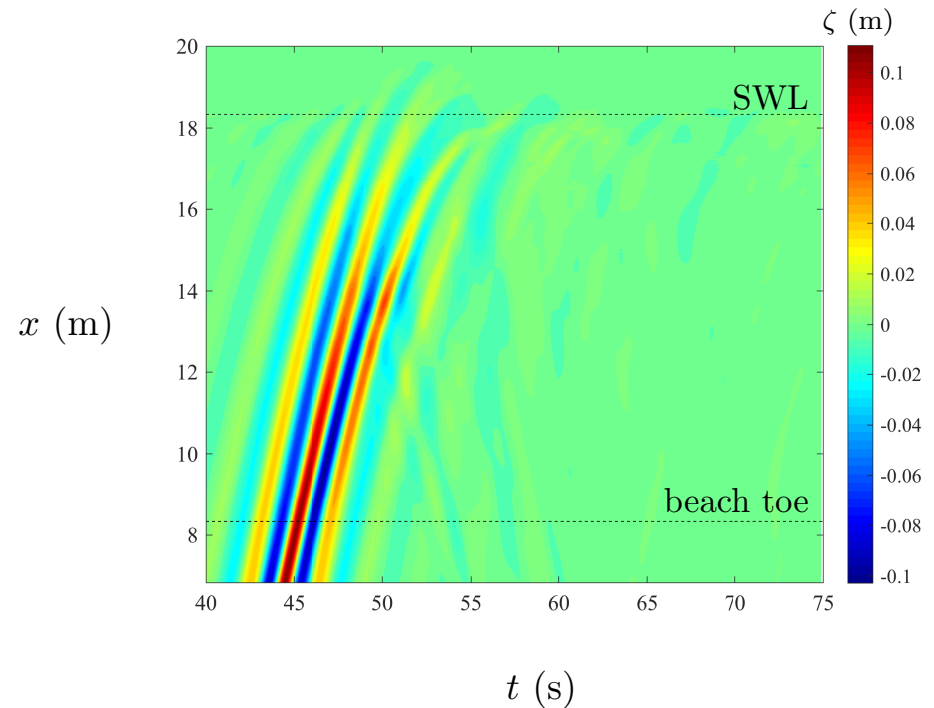

(a)

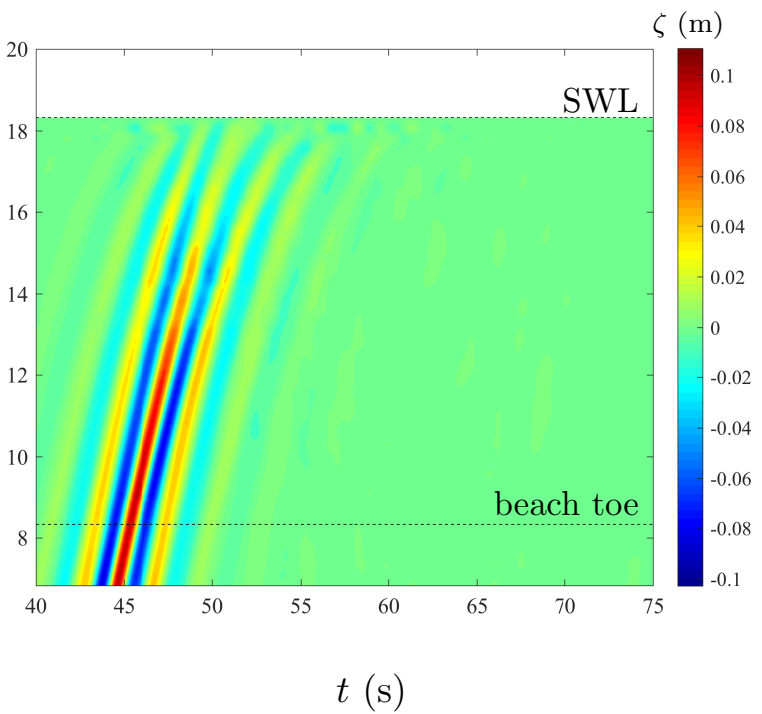

(b)

Figure 9: Space-time plots of NewWave run-up at a plane beach - Linear terms only, subtraction time series low-pass filtered at $1 \mathrm{~Hz}$ (WG17 and WG21): (a) numerical prediction, and (b) experimental gauge data, along basin centreline.

series. Therefore, some linear wave components with frequencies $<0.5 \mathrm{~Hz}$ are likely present in this Figure. Section 3.5 provides further analysis on long wave behaviour in the swash zone, without the complicating presence of the error wave.

Figure 12 presents a visualisation of the high frequency second-order harmonics. These sumfrequency harmonics include both bound and parasitic components. Error waves (denoted by $2^{+} \mathrm{e}$ ) are clearly seen trailing the super-harmonic bound waves $\left(2^{-} \mathrm{b}\right)$. Again there are reflections evident at $x \approx 13 \mathrm{~m}$ in the numerical results, whereas the experimental data show close to full absorption of these components by the beach.

The above analysis proves a useful tool by which to interpret the harmonic structure of the wave groups. The 2DH numerical model allows us to confirm the experimental findings of Hunt (2003), who found that the low frequency error waves from the $\pm 10^{\circ}$ and $\pm 30^{\circ}$ spread seas are approximately $2 / 3$ and $1 / 3$ respectively, of the size of the error wave produced in the uni-directional case. This is to be expected because of the lateral structure of the wave group: the displacement of fluid beneath an energetic group (the long bound wave) is coupled to the return flow beneath the group, which for spread sea cases can go sideways around the group as well as beneath it (and thereby drive longshore transient currents). Therefore, the impact on run-up prediction is not likely to be as severe. This is investigated further in Section 3.4.1.

It should be noted that because the fluid in the tank is water, formally, only the Navier-Stokes equations are appropriate for comparing experimental and numerical results. The paddle signals are derived either from linear theory, or in subsequent sections from the first two terms of a Stokes-type expansion of the solution to the potential flow equations. In contrast, the numerical scheme is based on a hybrid Boussinesq/NSWE model. Although it would be possible to derive a wave generation 'paddle' model for this equation set, we instead utilise the full potential flow 'paddle' motion in the numerical scheme. Therefore, given the number of approximations made in its construction, the numerical model works remarkably well.

\subsection{Wavelet analysis of focused wave group component interac- tions}

Wavelets are now used to gain further insight into the nonlinear behaviour of wave groups as they propagate up the beach. A continuous wavelet transform (CWT) is used to perform a timefrequency analysis of surface elevation data predicted by the numerical model. CWT scalograms 


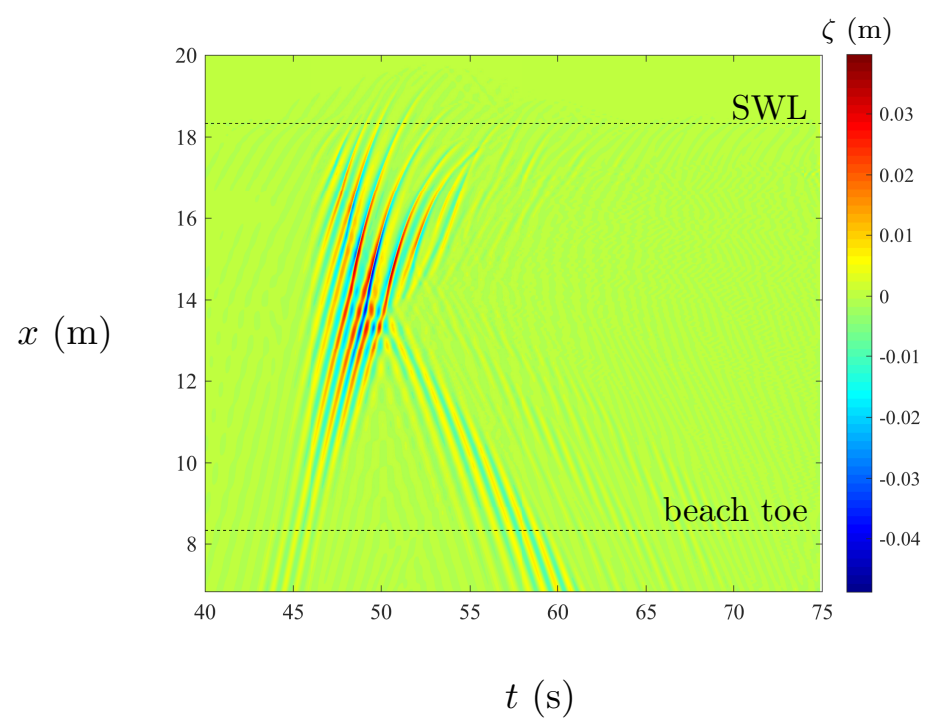

(a)

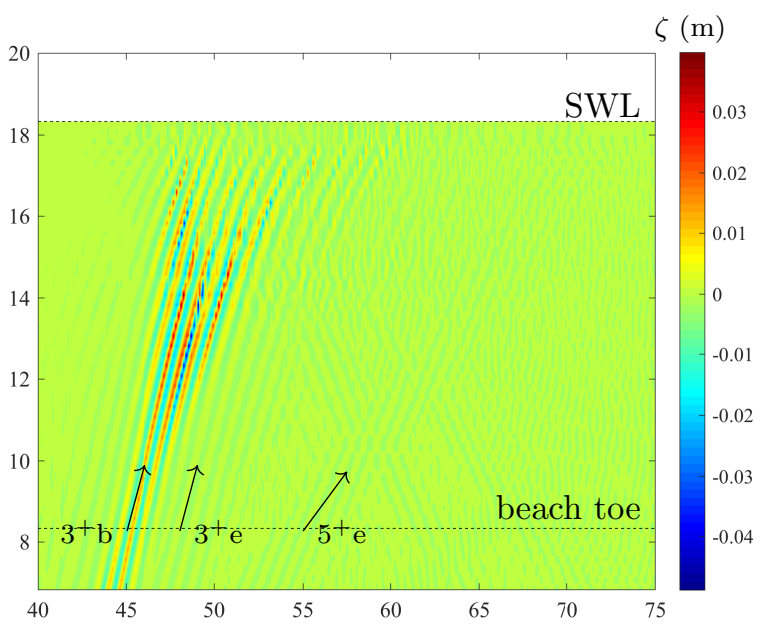

$t(\mathrm{~s})$

(b)

Figure 10: Space-time plots of NewWave run-up at a plane beach - Odd higher order harmonics only, subtraction time series high-pass filtered at $1 \mathrm{~Hz}$ (WG17 and WG21): (a) numerical prediction, and (b) experimental gauge data, along basin centreline.

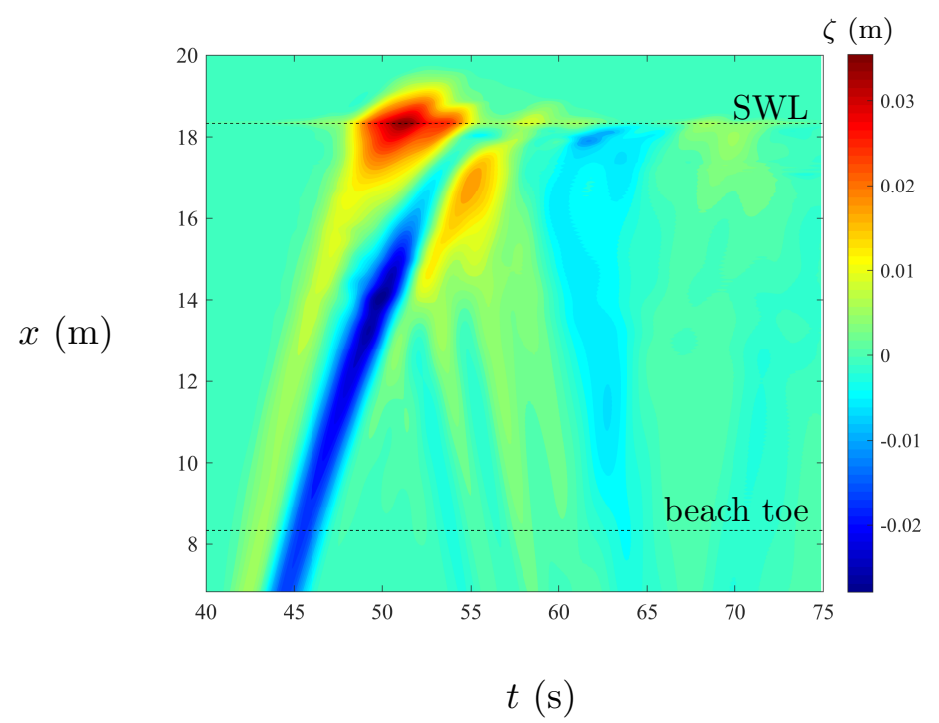

(a)

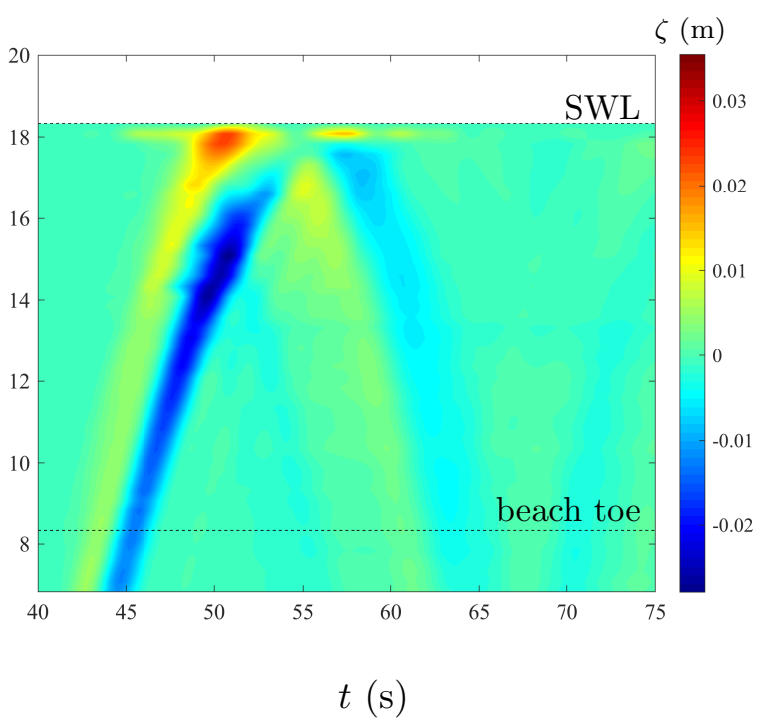

(b)

Figure 11: Space-time plots of NewWave run-up at a plane beach - Even harmonic, low frequency components only (i.e. second-order difference components or long waves), addition time series low-pass filtered at $0.5 \mathrm{~Hz}$ (WG17 and WG21): (a) numerical prediction, and (b) experimental gauge data, along basin centreline. 


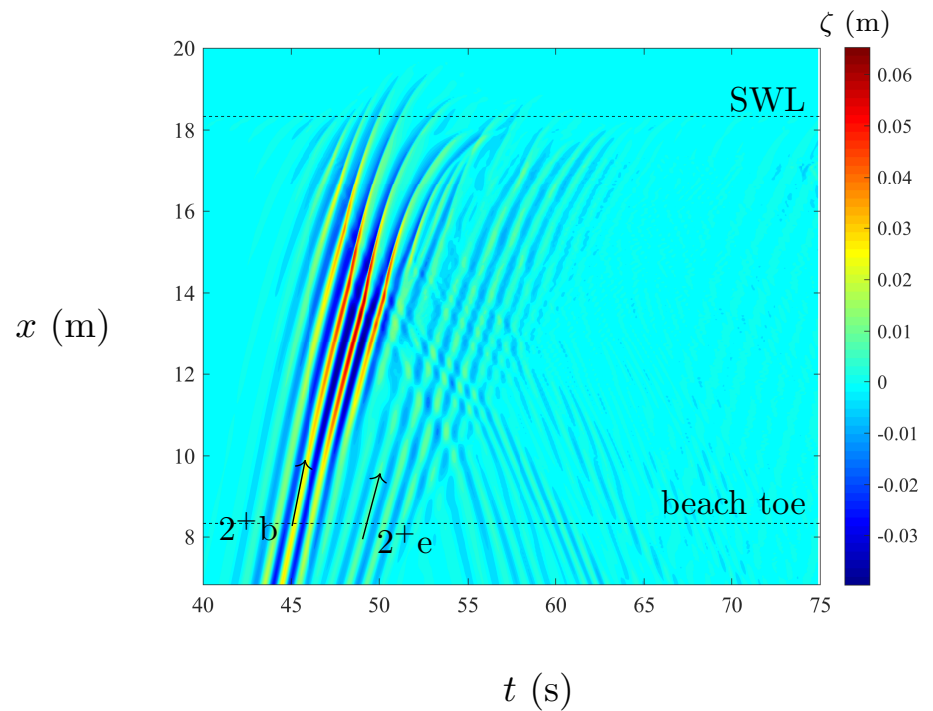

(a)

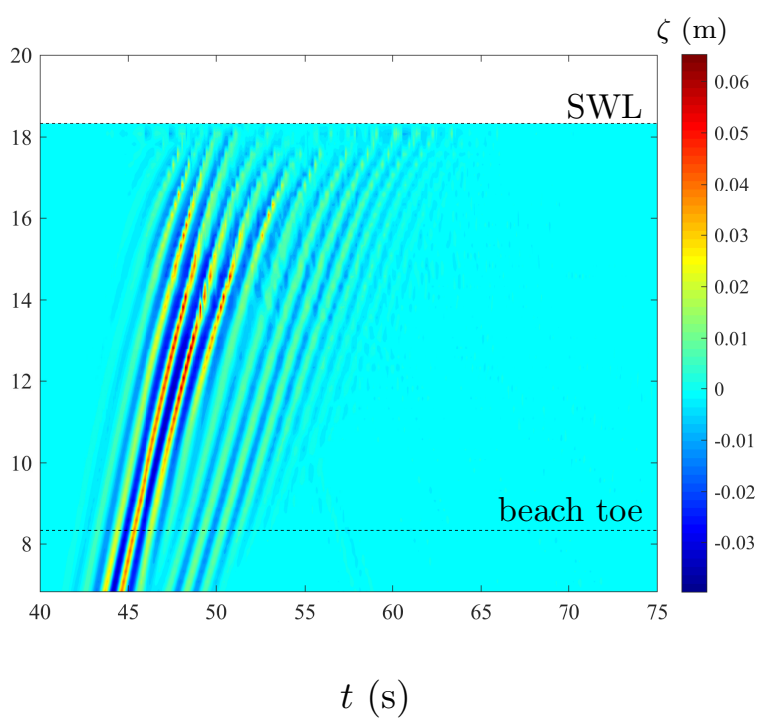

(b)

Figure 12: Space-time plots of NewWave run-up at a plane beach - Even harmonic, high frequency components only (second-order sum components), addition time series high-pass filtered at 0.5 Hz (WG17 and WG21): (a) numerical prediction, and (b) experimental gauge data, along basin centreline.

(the absolute values of the CWT as a function of time and frequency) illustrate the interactions of the wave group components as they propagate from offshore of the focus location through to the swash zone. The cone of influence is shown on the scalograms; outside the cone (in the grey regions) edge effects are significant. Wavelet transforms have previously been used by other researchers to analyse propagating waves, including Panizzo et al. (2002).

In the context of this analysis of free-surface wave fields, it should be remembered that any wavelet transform trades off frequency against time resolution, so some smearing of real features may occur and artificial artefacts may appear in the amplitude contour plots. Both of these will be affected by the choice of the mother wavelet. Here we use the simple Morlet wavelet which does not possess complete localisation in time but does preserve signal smoothness. We observe large and long lasting features in the analysis that correspond to robust properties of the underlying wave field. The wavelet analysis was performed on wavegroups generated numerically using both firstand second-order wavemakers. However, as there was remarkably little difference between the two, only the second-order results are presented here.

Figure 14 shows the wavelet results obtained from CWT analysis of the free surface elevation time series for WG17. The CWT scalograms are presented for 6 locations along the basin centreline between $x=2 \mathrm{~m}$ and $x=18 \mathrm{~m}$ to illustrate component interactions as the waves shoal and break. At $x=2 \mathrm{~m}$, the wavelet magnitude is maximum at a frequency of about $0.5 \mathrm{~Hz}$ coinciding with that of the linear components of the group; the asymmetry of the results indicates that the group has yet to come into focus when it reaches this location. Two 'spikes' (extending from 0.5 towards $2 \mathrm{~Hz}$ at times $t \approx 40$ and $43 \mathrm{~s}$ ) are crossed by a band at about $1 \mathrm{~Hz}$, the intersections corresponding to second-order super-harmonics, bound to the linear wave group. At $x=8.33 \mathrm{~m}$, focusing is evident from the symmetry of the scalogram. Here, the energies of the linear and bound superharmonic wave components appear exactly in phase at $t \approx 45 \mathrm{~s}$, with maxima at about 0.5 and 1 $\mathrm{Hz}$ respectively. A faint blue patch can be seen at $t \approx 55 \mathrm{~s}$ and $f \approx 0.5 \mathrm{~Hz}$, perhaps corresponding to a very low amplitude linear wave component that has been reflected from the beach. The light blue patches at about $1 \mathrm{~Hz}$ that occur at $t \approx 50$ and $58 \mathrm{~s}$ are most likely due to trailing secondorder super-harmonic components arising as error waves and reflected second-order super-harmonic components arriving back from the beach respectively. We note that the corrected paddle signal was designed to eliminate the second order sub-harmonic error wave only, and as such the sum frequency waves were unaffected. As the wave group propagates further up the beach it starts to defocus, before breaking and forming a series of bores. At $x=12 \mathrm{~m}$, the linear wave components 
at $f \approx 0.5 \mathrm{~Hz}$ have most energy, and the bound second-order super-harmonics are visible at about $t \approx 47$ and $49 \mathrm{~s}$.

At $x=15 \mathrm{~m}$, where the waves are beginning to break, the magnitude of the $0.5 \mathrm{~Hz}$ linear wave component has decreased as wave breaking sheds energy to higher frequencies. The bound super-harmonics contain energy across a range of frequencies up to about $10 \mathrm{~Hz}$, and appear to have distinct energy intersections at second-, and third-order super-harmonics. The peak energy of the bound super-harmonics occurs at $48.5 \mathrm{~s}$ for the second-order components $(1 \mathrm{~Hz})$. Reflected super-harmonics can be seen at $t \approx 53.5 \mathrm{~s}$ and $56 \mathrm{~s}$. A reflected second-order sub-harmonic below $f \approx 0.3 \mathrm{~Hz}$ is also evident as a weak structure at about $54 \mathrm{~s}$. Seiching motions may also be present, indicated by the energy content between 0.1 and $0.3 \mathrm{~Hz}$. At $x=17 \mathrm{~m}$, the scalograms are complicated by reflections and nonlinear interactions in the swash zone. Again the linear component is predominant at $0.5 \mathrm{~Hz}$, but with its energy spread between about 47 and $54 \mathrm{~s}$. There appears to be a reflected component at about 53.5 to $55 \mathrm{~s}$, which may be diffused in the breaker zone. The leading super-harmonic bound components at $t \approx 47.5 \mathrm{~s}$ have significant energy between 1 and $>2 \mathrm{~Hz}$, with peaks at $\approx 1 \mathrm{~Hz}$ (second-order) and $\approx 1.5 \mathrm{~Hz}$ (third-order). The second-order super-harmonic component also seems to have substantial energy spread, i.e. the yellow area at $\approx 1 \mathrm{~Hz}$ from $t \approx 47.5$ to $50.5 \mathrm{~s}$ (at which time a second 'spike' of bound super-harmonics arrives). Reflected super-harmonic components can be discerned at $t \approx 53$ and $55 \mathrm{~s}$, with energy at the higher harmonics and peaks respectively at $f \approx 1.4 \mathrm{~Hz}$ (third-order) and $f \approx 1.8 \mathrm{~Hz}$ (fourth-order). Further energy can be seen at $t \approx 58 \mathrm{~s}$ spread across frequencies from 1 to $2 \mathrm{~Hz}$. This is most likely a consequence of wave-wave interactions in the breaking zone, possibly very small re-reflections from the breaker line (so perhaps an artefact of the breaking criterion). A relative increase in low-frequency energy (between 0.15 and $0.3 \mathrm{~Hz}$ ) is visible at $t \approx 50$ to $55 \mathrm{~s}$. At $x=18 \mathrm{~m}$, very close to the shoreline, the incident and reflected components almost coincide. Here, the scalograms are similar to those at $x=17 \mathrm{~m}$, except that the linear wave and its reflection have a peak at $f \approx 0.5 \mathrm{~Hz}$ centred at $t \approx 50 \mathrm{~s}$ and covering a range from 46.5 to $52 \mathrm{~s}$. The sub-harmonic content at the lowest frequencies is much stronger, probably because of resonance in the swash zone. There are several spikes relating to super-harmonic components: one at $t \approx 46.5 \mathrm{~s}$ bound to the incoming linear waves; another at $t \approx 48 \mathrm{~s}$ the superposition of a second set of super-harmonic components bound to the incoming linear waves and the first set of reflected super-harmonics; and a third spike at $t \approx 51.5 \mathrm{~s}$ related to the second set of outgoing reflected super-harmonics. Second-, third-, and fourth-order super-harmonics are predominant, though there appears to be wave energy at frequencies up to 10 Hz. Lastly, there is energy at $t \approx 55 \mathrm{~s}$, spread across super-harmonic frequencies from second- to fourth-order and beyond, which may have arisen from wave-wave interactions in the surf zone.

\subsection{Run-up analysis of focused wave groups}

Accurate calculation of maximum run-up caused by extreme waves is essential for the safe siting of new developments at the coast, or to assess the flood risk posed to existing infrastructure. Run-up is defined here as the instantaneous vertical elevation of the water surface above still water level on the surface of the beach. In the UKCRF tests described above, run-up was measured visually by filming the swash zone. Hunt (2003) estimated the error in these measurements to be $\pm 10 \mathrm{~cm}$ along the slope, which corresponds to a vertical measurement of $\pm 5 \mathrm{~mm}$ for a 1:20 beach.

We now consider simulations of several of the UKCRF wave group experiments used to estimate maximum run-up. The wave groups chosen are those with the largest linear focus amplitude $\left(A_{f}\right)$ for each focus location, namely the beach toe, $\frac{3}{4}$ offshore depth, and $\frac{1}{2}$ offshore depth. Multiple spread angles are considered. Table 2 summarises the measured and predicted run-up results for each of the wave groups. As expected, the largest run-up events are produced by the uni-directional wave groups, the largest of all being WG5, the trough-focused wave group focusing at $\frac{3}{4}$ offshore depth. In all cases (both numerical and experimental), the trough-focused wave groups produce larger run-up than their crest-focused counterparts. As the spread angle of the incident wave group increases, the maximum run-up decreases. For a given spread angle, with a linear focus amplitude $A_{f}=114 \mathrm{~mm}$, the maximum run-up is generally very similar when the focus location is the beach toe or $\frac{3}{4}$ offshore depth. The only major departure from this is for uni-directional wave groups 

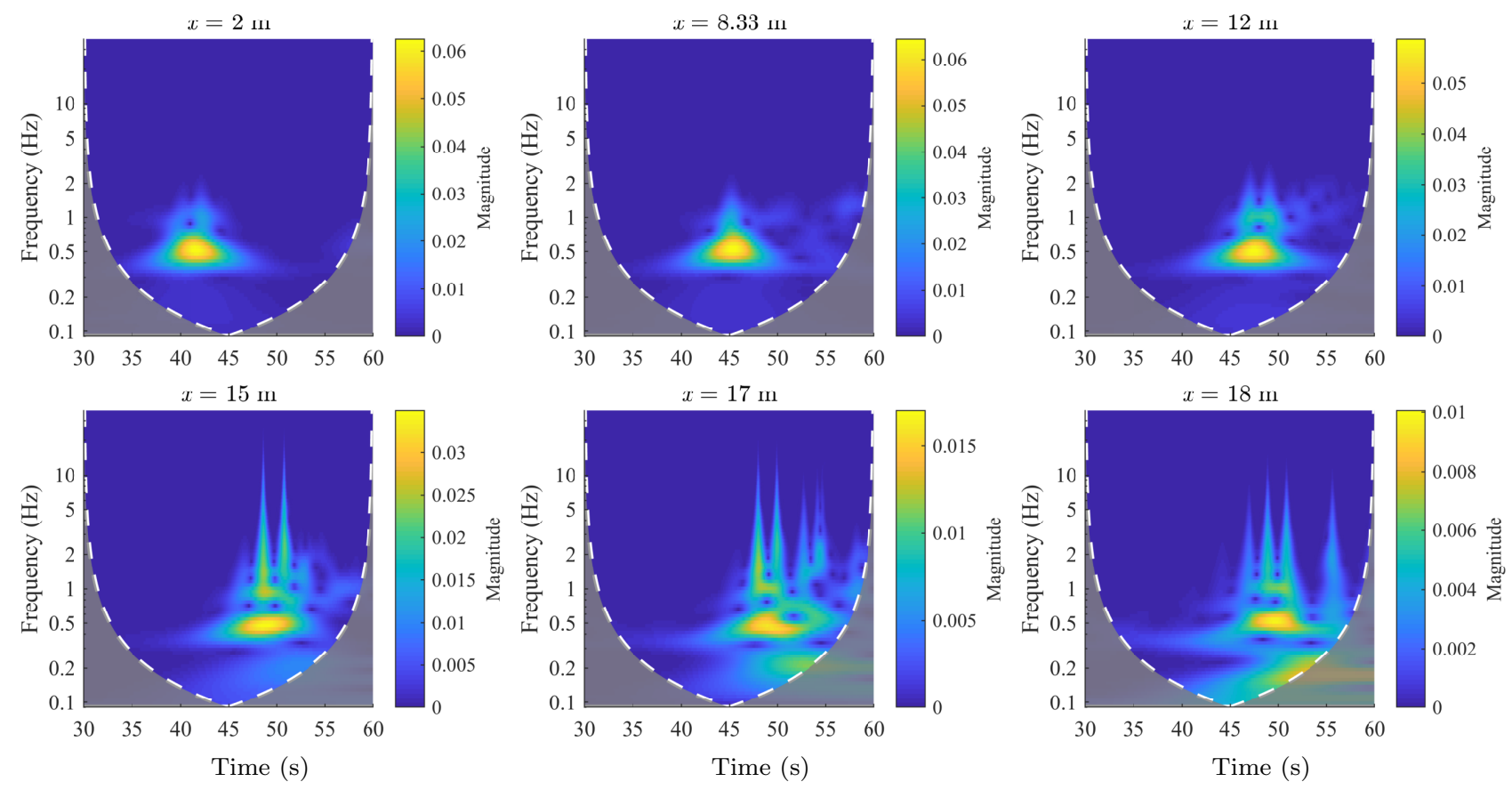

Figure 13: CWT scalograms for free surface elevation time series of $\pm 30^{\circ}$ spread, crest-focused, multi-directional wave group WG17 at the following locations along basin centreline: $x=2,8.33,12,15,17$ and $18 \mathrm{~m}$.

WG1 and WG2, where the $\frac{3}{4}$ offshore depth focus location produces a significantly larger run-up event in both the laboratory and numerical results.

In general, there is good agreement between the UKCRF measurements and the numerically predicted values. The largest magnitude error is for wave group WG17 at 16.9\%, followed by WG30 at $-15 \%$. Inspection of Table 2 reveals that the numerical model tends to over-predict the run-up of crest-focused wave groups, whereas the trough-focused wave group run-up is generally underpredicted. Table 3 presents the calculated percentage bias in the run-up results. The percentage bias measures the average tendency of the predicted values to be larger or smaller than the corresponding observations, and is given by

$$
\% \mathrm{BIAS}=100\left(\frac{\sum\left(R_{p}-R_{m}\right)}{\sum R_{m}}\right)
$$

where $R_{m}$ is the measured run-up, and $R_{p}$ is the predicted run-up from Table 2 . Whereas the total percentage bias of all wave groups is $2.2 \%$, individual calculations for both crest- and trough-focused wave groups reveal $+8.3 \%$ bias for the crest-focused groups, and $-2.4 \%$ bias for the trough-focused groups.

Table 4 lists the predicted run-up values for the directionally spread wave groups, expressed as a percentage of the run-up of the corresponding uni-directional wave group with the same linear focus amplitude and focus location. Grouping the main wave group types together (by spread angle and phase), and calculating the average percentage leads to Table 5, which presents a comparison with Hunt's measurements. Hunt reports an approximate $20 \%$ reduction in run-up for a $\pm 10^{\circ}$ spread sea, and a $40 \%$ reduction for a $\pm 30^{\circ}$ spread sea. The reductions in run-up predicted by the numerical model are in general agreement with the values obtained by Hunt, although with slightly larger reductions observed, particularly for the $\pm 10^{\circ}$ spread wave groups.

The values of the tuning parameters are likely to play a significant part in controlling the accuracy of results obtained using the present model, particularly the value of $C_{f}$ in the swash zone. Judge et al. (2018) apply a number of different methods for calculating the eddy viscosity and bed friction coefficient using the present numerical model to carry out simulations involving nearshore circulation. They conclude that a more sophisticated turbulence model may improve representation of the hydrodynamics in the swash zone, and thus have an impact on numerical predictions of run-up. The model does not permit wave reformation from bores owing to the rather 


\begin{tabular}{llllllll}
\hline $\begin{array}{l}\text { Wave group } \\
\text { ref. }\end{array}$ & $\begin{array}{l}A_{f} \\
(\mathrm{~mm})\end{array}$ & $\begin{array}{l}\text { Spread } \\
\text { angle }\end{array}$ & $\begin{array}{l}\text { Phase } \\
(\mathrm{rad})\end{array}$ & $\begin{array}{l}\text { Focus } \\
\text { location }\end{array}$ & $\begin{array}{l}R_{m} \\
(\mathrm{~mm})\end{array}$ & $\begin{array}{l}R_{p} \\
(\mathrm{~mm})\end{array}$ & $\begin{array}{l}\% \text { error } \\
\left(R_{p} \mathrm{v} R_{m}\right)\end{array}$ \\
\hline WG1 & 114 & $0^{\circ}$ & 0 & beach toe & 98 & 109 & 11.2 \\
WG2 & 114 & $0^{\circ}$ & 0 & $\frac{3}{4}$ depth & 110 & 117 & 6.4 \\
WG3 & 90 & $0^{\circ}$ & 0 & $\frac{1}{2}$ depth & 90 & 97 & 7.8 \\
WG5 & 114 & $0^{\circ}$ & $\pi$ & beach toe & 135 & 138 & 2.2 \\
WG6 & 114 & $0^{\circ}$ & $\pi$ & $\frac{3}{4}$ depth & 136 & 137 & 0.7 \\
WG7 & 90 & $0^{\circ}$ & $\pi$ & $\frac{1}{2}$ depth & 115 & 113 & -1.7 \\
WG17 & 114 & $\pm 30^{\circ}$ & 0 & beach toe & 59 & 69 & 16.9 \\
WG18 & 114 & $\pm 30^{\circ}$ & 0 & $\frac{3}{4}$ depth & 60 & 65 & 8.3 \\
WG19 & 90 & $\pm 30^{\circ}$ & 0 & $\frac{1}{2}$ depth & 51 & 56 & 9.8 \\
WG21 & 114 & $\pm 30^{\circ}$ & $\pi$ & beach toe & 76 & 72 & -5.3 \\
WG22 & 114 & $\pm 30^{\circ}$ & $\pi$ & $\frac{3}{4}$ depth & 75 & 74 & -1.3 \\
WG23 & 90 & $\pm 30^{\circ}$ & $\pi$ & $\frac{1}{2}$ depth & 65 & 63 & -3.1 \\
WG25 & 114 & $\pm 10^{\circ}$ & 0 & beach toe & 79 & 85 & 7.6 \\
WG26 & 114 & $\pm 10^{\circ}$ & 0 & $\frac{3}{4}$ depth & 85 & 86 & 1.2 \\
WG27 & 90 & $\pm 10^{\circ}$ & 0 & $\frac{1}{2}$ depth & 70 & 76 & 8.6 \\
WG29 & 114 & $\pm 10^{\circ}$ & $\pi$ & beach toe & 106 & 107 & 0.9 \\
WG30 & 114 & $\pm 10^{\circ}$ & $\pi$ & $\frac{3}{4}$ depth & 107 & 91 & -15.0 \\
WG31 & 90 & $\pm 10^{\circ}$ & $\pi$ & $\frac{1}{2}$ depth & 86 & 84 & -2.3 \\
\hline
\end{tabular}

Table 2: Measured and predicted run-up of focused wave groups at a plane beach in the UKCRF.

\begin{tabular}{lc}
\hline Wave groups & \% BIAS \\
\hline All & 2.2 \\
Crest-focused & 8.3 \\
Trough-focused & -2.4 \\
\hline
\end{tabular}

Table 3: \% bias of run-up results

simple way wave breaking is treated. The foregoing warrant further research. A higher resolution computational grid might also lead to improved results; however achieving this for the spread-sea case would require more computational power than was available for the present research study.

It should be noted that a recent study on uni-directional wave group optimisation on a similar 1:20 beach (Whittaker et al., 2017) has shown that both the focus position $\left(x_{f}\right)$ and the phase $(\phi)$ of the wave at focus are key parameters for determining run-up. The 'stripes' in Figure 10 of Whittaker et al. correspond to optimal combinations of focus location and phase causing maximum run-up. In contrast, for $x_{f}$ fixed with $\phi$ changing, the run-up can change from its local maximum to minimum rather quickly, with very sharp minima and rather broader maxima. In the uni-directional simulations, the run-up could change with phase by close to a factor of 2. Comparable effects should be expected in directionally spread groups, but full investigation of this is left for future work.

\subsubsection{Effect of long error wave on NewWave run-up}

Orszaghova et al. (2014) calculated second-order paddle signals for uni-directional focused wave groups at the UKCRF (WG1-WG8), which almost completely eliminated the low-frequency error wave. The error wave is not fully eliminated because Schäffer's second order wave generation theory (Schäffer, 1996) is for potential flow, and is only approximately correct for the Madsen and Sørensen Boussinesq equations. In the present research, the multi-directional wave groups WG17 and WG21 are again considered, using similarly corrected paddle signals. The aim here is to confirm that the long error wave travelling ahead of the wave group is largely eliminated, and to determine the impact this has on the maximum run-up of the multi-directional UKCRF tests. Although highfrequency error waves are still generated, these arrive at the beach after the main wave group and 


\begin{tabular}{ccccc}
\hline & & \multicolumn{3}{c}{ \% of uni-directional } \\
WG & Spread & Phase & $R_{p}$ & Ref. WG \\
\hline WG17 & $\pm 30^{\circ}$ & 0 & 63.3 & WG1 \\
WG18 & $\pm 30^{\circ}$ & 0 & 55.6 & WG2 \\
WG19 & $\pm 30^{\circ}$ & 0 & 57.7 & WG3 \\
WG21 & $\pm 30^{\circ}$ & $\pi$ & 52.2 & WG5 \\
WG22 & $\pm 30^{\circ}$ & $\pi$ & 54.0 & WG6 \\
WG23 & $\pm 30^{\circ}$ & $\pi$ & 55.8 & WG7 \\
WG25 & $\pm 10^{\circ}$ & 0 & 78.0 & WG1 \\
WG26 & $\pm 10^{\circ}$ & 0 & 73.5 & WG2 \\
WG27 & $\pm 10^{\circ}$ & 0 & 78.4 & WG3 \\
WG29 & $\pm 10^{\circ}$ & $\pi$ & 77.5 & WG5 \\
WG30 & $\pm 10^{\circ}$ & $\pi$ & 66.4 & WG6 \\
WG31 & $\pm 10^{\circ}$ & $\pi$ & 74.3 & WG7 \\
\hline
\end{tabular}

Table 4: Predicted run-up of directionally spread wave groups expressed as a percentage of the run-up of the corresponding uni-directional wave groups with the same linear focus amplitude and focus location (Ref. WG) at a plane beach in the UKCRF.

\begin{tabular}{lcc}
\hline & $\begin{array}{c}\text { mean predicted } \\
\alpha_{R}\end{array}$ & $\begin{array}{c}\text { mean \% measured } \\
\alpha_{R} \text { (Hunt, 2003) }\end{array}$ \\
\hline $\pm 10^{\circ}$ crest focus & 76.6 & 80.2 \\
$\pm 10^{\circ}$ trough focus & 72.8 & 78.3 \\
$\pm 30^{\circ}$ crest focus & 58.9 & 59.8 \\
$\pm 30^{\circ}$ trough focus & 54.0 & 56.3 \\
\hline
\end{tabular}

Table 5: Spread sea run-up expressed as a percentage of uni-directional run-up for both numerically predicted and measured values. 


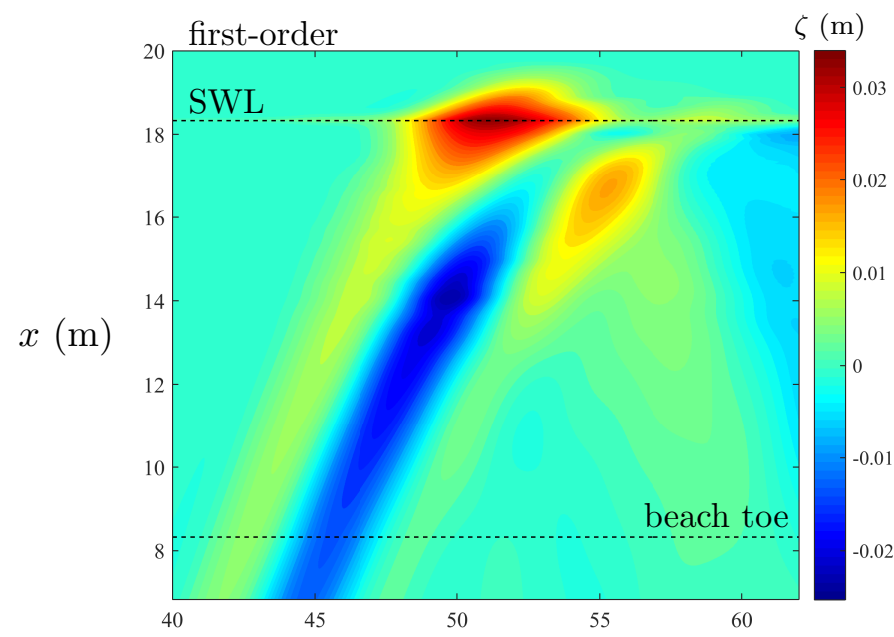

$t(\mathrm{~s})$

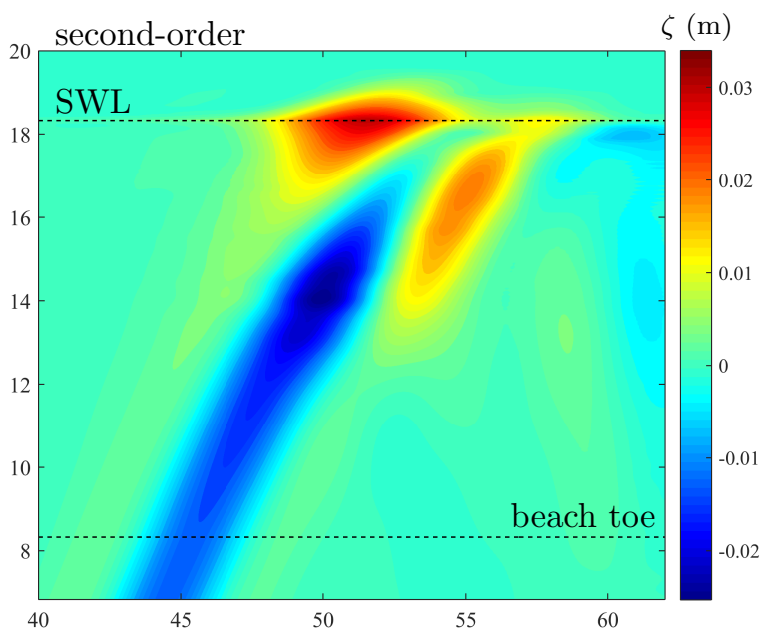

$t(\mathrm{~s})$

(b)

Figure 14: Space-time plots of multidirectional NewWave run-up at a plane beach - long waves only (low-pass filtered at $0.3 \mathrm{~Hz})$, WG17 and WG21 ( $\pm 30^{\circ}$ spread). Model predictions along basin centreline using (a) first order paddle signals, and (b) second-order paddle signals.

so do not interfere with the maximum run-up.

The same method of separation of harmonics applied in the previous section is again implemented here, to isolate the second-order low-frequency components of the wave groups in order to identify whether contaminating long error waves are successfully removed. This time, the addition time series is low-pass filtered at $0.3 \mathrm{~Hz}$, to remove the effect of out-of-phase linear components closer to the shore. Figure 15 compares the result of this exercise with the original numerical simulation of wave groups WG17 and WG21 generated with the first-order paddle signal. The figure shows reduction in the amplitude of the wave crest leading the main wave group, indicating the removal of the free long error wave. A small hump remains that increases in amplitude as the wave group shoals, along with the amplitudes of both the set-down and the trailing long wave crest. The effect of the second-order correction on the run-up is quantified in Table 6. A reduction in runup of $14.5 \%$ was observed for the crest-focused wave group, whereas a $13.9 \%$ reduction was observed for the trough-focused case.

A corresponding exercise is performed for wave groups WG25 and WG29 (with a spread angle of $\pm 10^{\circ}$ ), and the results are presented in Figure 16. The numerical simulation using first-order paddle signals confirms the presence of a larger low-frequency error wave than was observed for the wave groups with a spread angle of $\pm 30^{\circ}$. The application of a second-order correction to the paddle signals again largely removes the long error wave, and leads to a deeper set down beneath the main wave group. Whereas a significant difference in maximum run-up was observed between the crest (WG25) and trough-focused (WG29) wave groups using first-order wave generation, this difference is almost eradicated when using second-order wave generation (reductions of $20 \%$ and $37.3 \%$ respectively), resulting in similar run-up predictions for these wave groups (Table 6 ). This is similar to the findings of Orszaghova et al. (2014) in relation to uni-directional wave groups WG1 and WG5. It is likely that the long error wave interacts with the leading waves of trough-focused wave groups in a way that causes greater amplification of run-up than for crest-focused groups, and therefore its removal has the effect of narrowing the gap between the maximum run-up of both sets of groups. However, further research is required to investigate this behaviour.

Figure 17 presents a comparison between the numerically predicted free surface elevation distributions in the wave basin at maximum run-up for WG25 using first- and second-order paddle signals. It can clearly be seen that the second-order correction results in a significant reduction in the extent, in both the longshore and cross-shore directions, of the maximum run-up event. It should be noted that the second-order correction has been applied in the numerical model only-new 


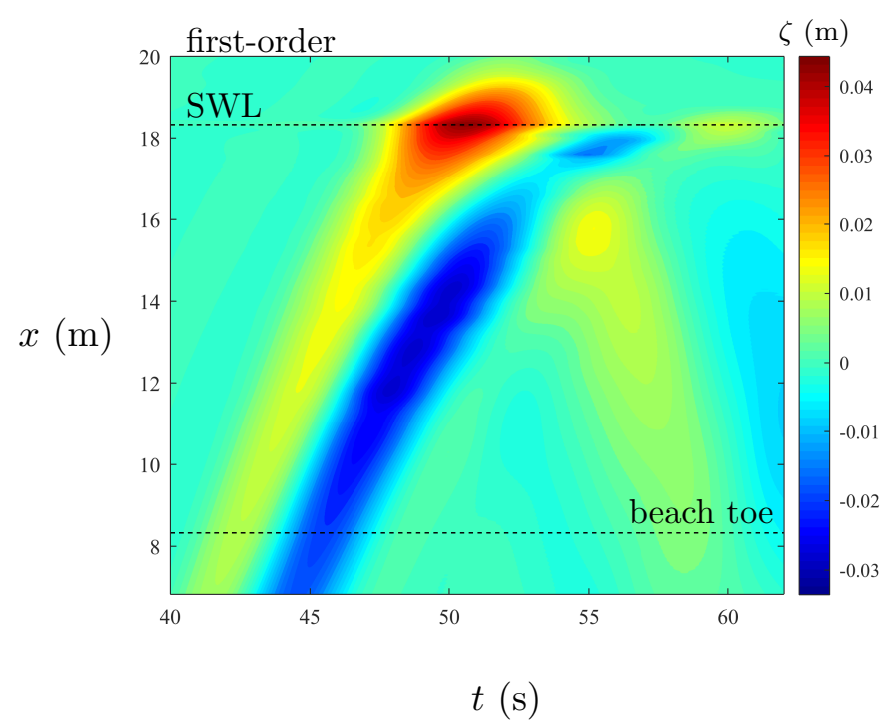

(a)

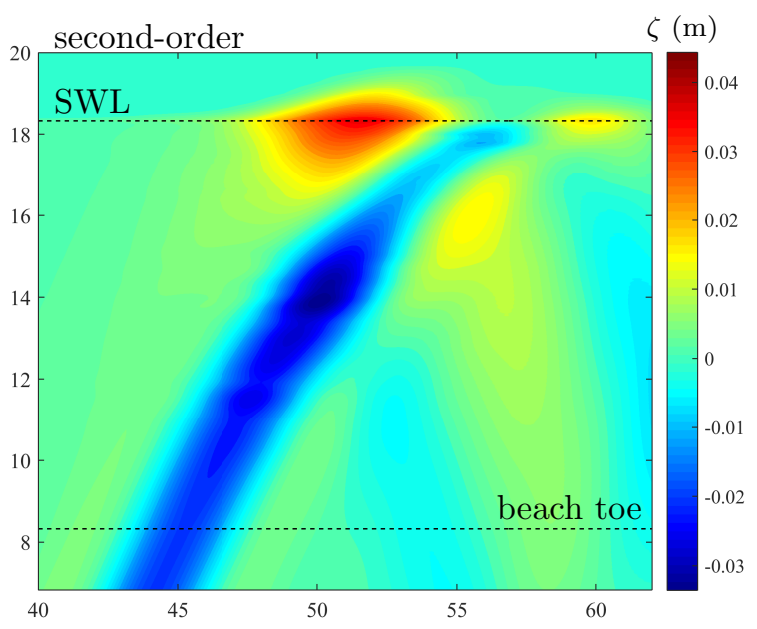

$t(\mathrm{~s})$

(b)

Figure 15: Space-time plots of multidirectional NewWave run-up at a plane beach - long waves only (low-pass filtered at $0.3 \mathrm{~Hz}), \mathrm{WG} 25$ and WG29 $\left( \pm 10^{\circ}\right.$ spread). Model predictions along basin centreline using (a) first order paddle signals, and (b) second-order paddle signals.

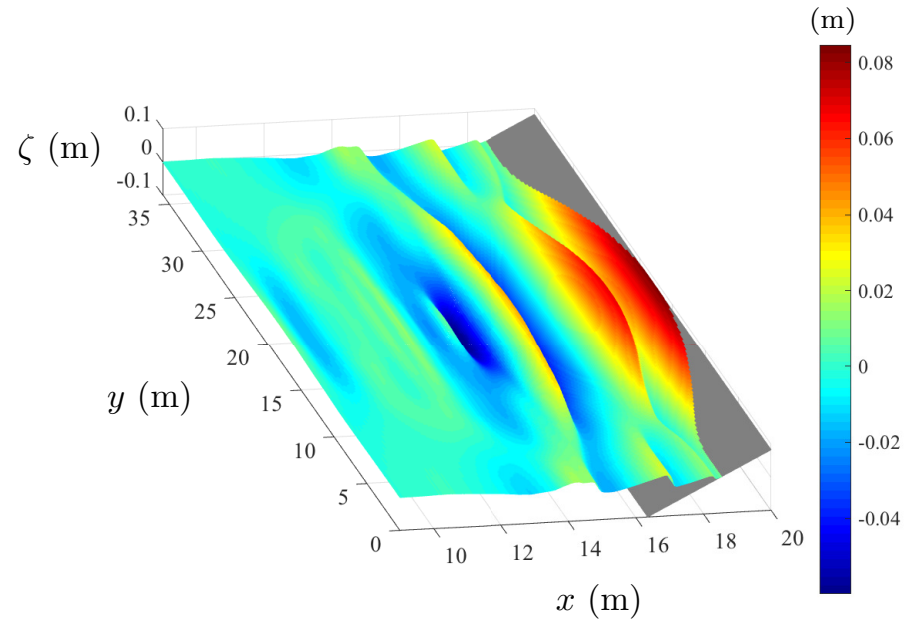

(a)

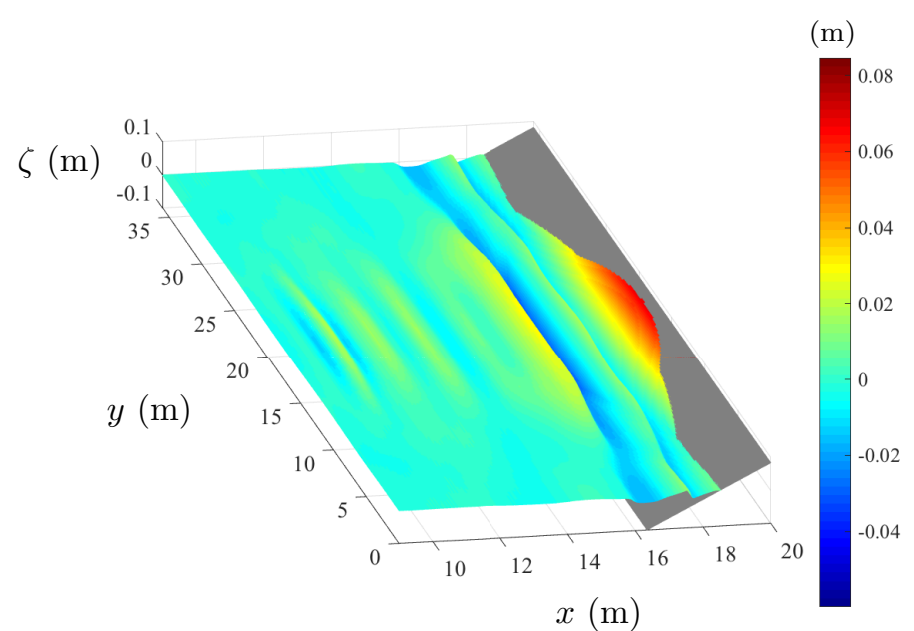

(b)

Figure 16: Numerical predictions of surface elevation at the time of maximum run-up for (a) WG25 $\left( \pm 10^{\circ}\right.$ spread) using first-order paddle signals; and (b) WG25 using second-order paddle signals. 


\begin{tabular}{cccc}
\hline & $\begin{array}{c}R_{p} \\
(\mathrm{~mm})\end{array}$ & $\begin{array}{c}R_{p, \text { corrected }} \\
(\mathrm{mm})\end{array}$ & \% difference \\
\hline WG17 & 69 & 59 & $-14.5 \%$ \\
WG21 & 72 & 62 & $-13.9 \%$ \\
WG25 & 85 & 68 & $-20.0 \%$ \\
WG29 & 107 & 67 & $-37.4 \%$ \\
\hline
\end{tabular}

Table 6: Effect of second-order correction on numerically predicted NewWave run-up.

experiments with second-order wave generation have not been carried out. Therefore, applying the second-order correction does not improve the agreement between the numerical and experimental data, the latter resulting from first-order paddle signals.

Orszaghova et al. (2014) found, that by implementing second-order wave generation on unidirectional focused wave groups, predicted values of run-up were on average $40 \%$ smaller for the case considered than when first-order paddle signals were used. As demonstrated by the present study, the presence of an error wave due to first-order paddle signals also has an impact on the run-up of multi-directional wave groups, although as expected, the effect becomes less significant with increasing angle of spread. This is likely due to two effects: the reduction of the size of the bound set-down due to directional spreading; and the spreading out of the long error wave laterally.

\subsection{Long wave evolution}

The numerical generation of waves correct to second-order in 2DH provides us with the opportunity to study propagation of the long waves associated with multi-directional wave groups, with minimal impact from the free long error wave. Using numerical data for second-order wave groups WG25 $\left( \pm 10^{\circ}\right.$ spread $)$ and WG17 $\left( \pm 30^{\circ}\right.$ spread $)$, the surface elevation time series along the basin centreline is low-pass filtered at $0.3 \mathrm{~Hz}$, and the results are presented in Figure 18. In Figure 18 (a), small crests are visible either side of the bound set-down (blue) beneath WG25, as the waves propagate away from the paddles. The set-down deepens as the wave group shoals, reaching a local minimum at $x \approx 14 \mathrm{~m}$, and $t \approx 50 \mathrm{~s}$. The long wave travelling ahead of the set-down increases in amplitude rapidly from $x>16 \mathrm{~m}$, as radiation stress gradients in the surf zone generate a dynamic setup, leading to a low-frequency run-up motion above the SWL, which results in the reflection of a free wave crest offshore. The interaction between this reflected crest and the incoming set-down generates a transient node, similar to that noted by Lara et al. (2010) although more short-lived, lasting approximately $2 \mathrm{~s}$. The reflected crest is preceded by a shallow long wave trough that appears to originate from the breakpoint (identifiable in the total free surface plots, not shown). Breakpoint forced waves are long waves forced by oscillations in the position of the break point; they were first theorised by Symonds et al. (1982), and subsequently by Schäffer (1993a) and others. Experiments by Baldock (2006) involving normally incident wave groups interacting with a 1:10 beach confirmed the generation of breakpoint forced waves. RANS modelling by Lara et al. (2010) of a uni-directional transient wave group propagating over a discontinuous beach predicted similar behaviour. This research indicates that breakpoint forced waves can also be generated by multi-directional wave groups.

Figure 18 (b) presents results for the $\pm 30^{\circ}$ spread group (WG17). Compared with $\pm 10^{\circ}$ case, the bound set-down is shallower, and the low-frequency run-up motion at the shoreline is less severe. In contrast, the trailing incident wave crest experiences greater forcing as the wave group shoals, and continues to grow in the surf zone. Long wave offshore-traveling components are discernible, but much less significant than for the $\pm 10^{\circ}$ case. The source of some of these reflections can be traced to the breakpoint, potentially indicating the presence of breakpoint forced waves. The reduced long wave reflection (along the centreline) is due to sideways spreading of these components.

Figure ?? provides a 2DH visualisation of the long wave evolution of WG17. It shows how the long wave crest leading the set-down spreads in the longshore direction as it shoals, becoming partly trapped at the shoreline and creating oblique reflections. The incoming set-down grows in 
amplitude until $t=50 \mathrm{~s}$, after which the trapped leading long wave crest and its reflections forces it to spread laterally, losing amplitude as it does so. The loss of the linear waves due to breaking releases the long wave component trailing the main packet, which then rapidly grows locally as it shoals, travelling faster in the shallow water than the set-down ahead of it and causing a secondary low-frequency run-up event (at $t \approx 57 \mathrm{~s}$ ). The return flow is diverted sideways around the group.

\section{Conclusions}

A 2DH hybrid numerical model that implements enhanced Boussinesq equations pre-breaking and nonlinear shallow water equations post-breaking has been used to simulate the propagation of multidirectional wave groups in shallow water and their run-up at a plane beach. Excellent agreement has been obtained between the numerical predictions and previous laboratory measurements of free surface elevations for multi-directional NewWave focused wave group interactions with a plane beach in the UKCRF. Predicted and measured run-up values were generally in satisfactory agreement, though the numerical model tended to over-predict the run-up of crest-focused wave groups and under-predict the run-up of trough-focused wave groups.

The use of harmonic decomposition using phase-inverted pairs of multi-directional wave groups allowed much of the harmonic structure to be revealed, both components bound to the main groups and free error wave components released by the paddle with linear driver signals. The magnitude of the low-frequency error wave was found to be smaller for multi-directional wave groups than the corresponding uni-directional case. Its effect on run-up was found to be smaller than for an equivalent uni-directional focused wave group. The presence of second and third sum harmonic error waves created by the paddle did not affect peak run-up because these components arrive at the beach well after the main group. Wavelet analysis gave useful insights into the way in which the spectrum of wave components changed with time, and the influence of non-linearity on the resulting wave surface elevation distributions.

Low-pass filtering of the surface elevation time series for crest-focused multi-directional wave groups corrected to second order allowed further examination of the long wave evolution, including the generation of breakpoint forced waves in a spread sea. The lateral structure of the long waves in the surf and the swash zones has been revealed. The run-up and reflections caused by incident long waves become less severe with increasing spread angle, due to the longshore spreading of the low-frequency components.

This paper has shown that focused wave groups provide an ideal means of exploring the harmonic structure of waves in the coastal zone. The approach revealed explicitly the creation of spurious error waves by linear wavemakers, which might otherwise be concealed within random wave tests. The effect of linear as opposed to second-order accurate paddle motions is significant, both for unidirectional and directionally spread normally incident wave groups, in terms of maximum run-up on the beach.

These results are important because of the implications for the design of structures for coastal protection. A wave group corrected to second order represents a more realistic sea state, and thus produces more realistic run-up and overtopping events. Therefore, a numerical model that generates multi-directional waves correct to second order, could be used by the coastal engineer to design more efficient structures for coastal protection, and reduce the conservatism in seawall design. The present study and that of Orszaghova et al. (2014), confirm that second-order paddle motions are required when modelling run-up at laboratory-scale, and hence experiments using just linear theory may be significantly flawed. Just as for uni-directional waves (Fitzgerald et al., 2016; Whittaker et al., 2017), we propose that a localised, multi-directional focused wave group may be used as a 'design wave' for the simulation of run-up extremes in directionally spread seas. But this will require optimisation of both focus position and wave group phase at focus, as in Whittaker et al. (2017), and then comparison with random simulations for sea-states to provide robust extreme statistics, comparable to Fitzgerald et al. (2016). Both tasks are left for future work. 


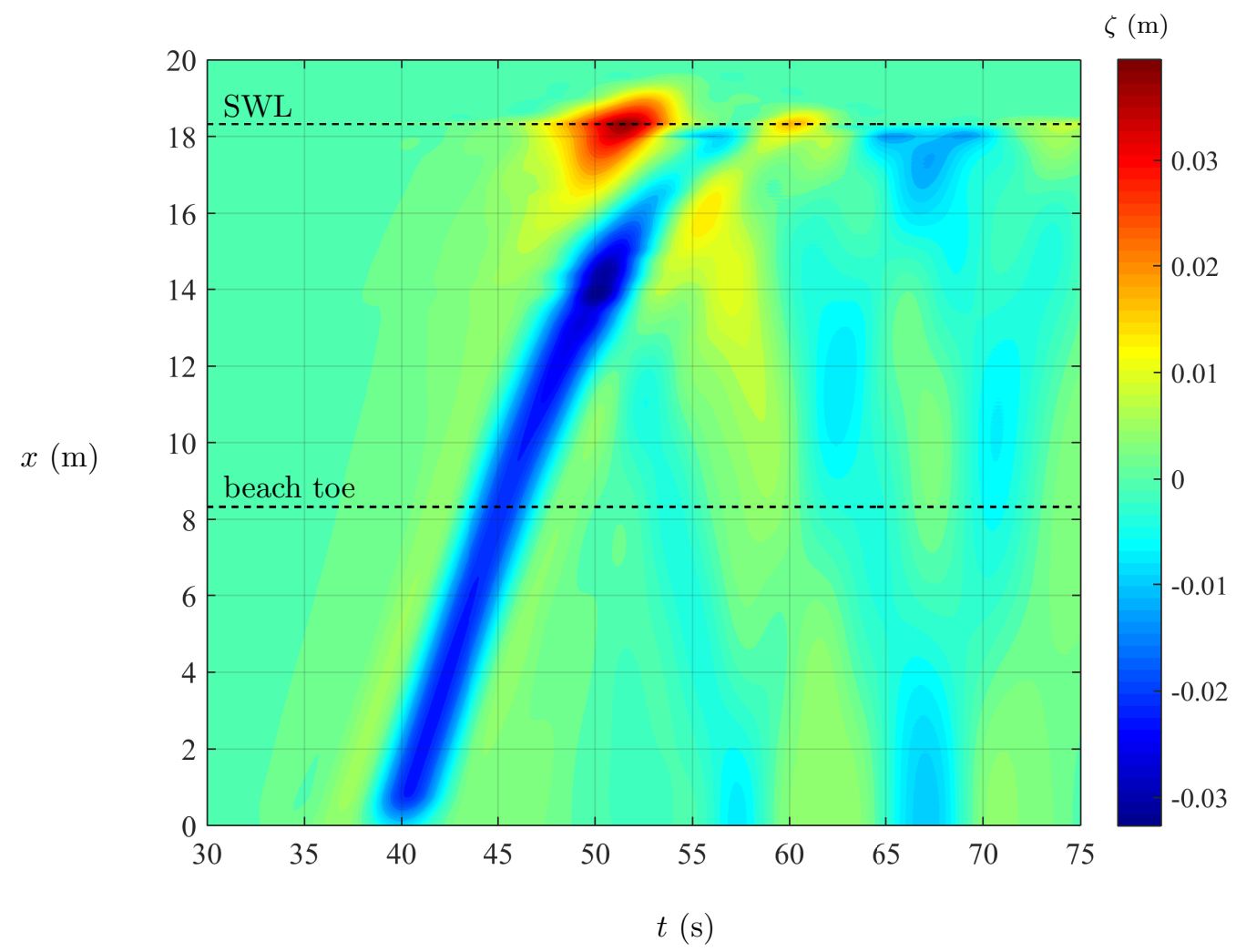

(a)

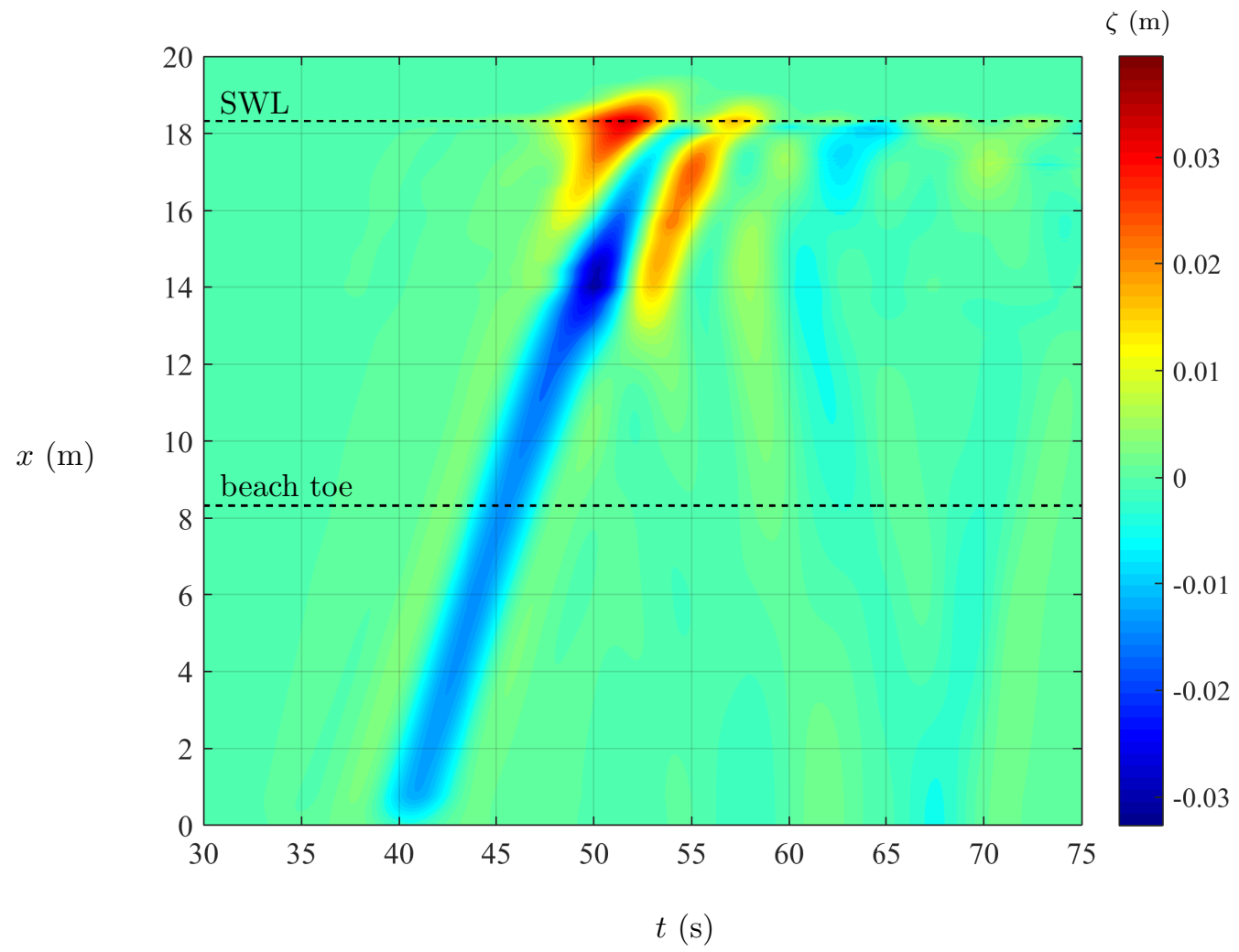

(b)

Figure 17: Space-time plots of (a) WG25 ( $\pm 10^{\circ}$ spread), and (b) WG17 ( $\pm 30^{\circ}$ spread), low-pass filtered at 0.3 Hz. Model predictions along basin centreline using second-order paddle signals. 

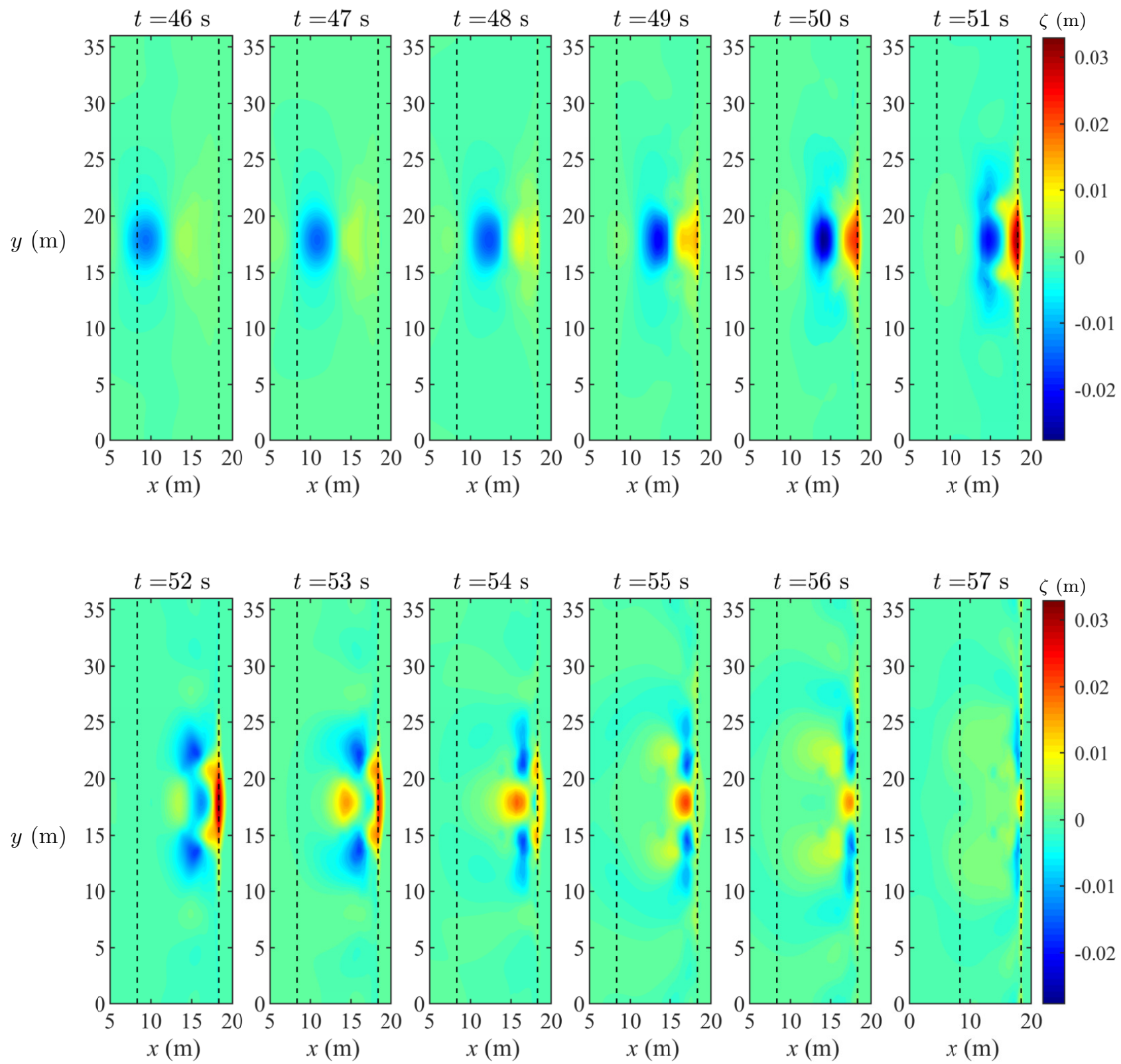

Figure 18: Overhead (2DH) view of long wave evolution for WG17 $\left( \pm 30^{\circ}\right.$ spread), calculated from second-order paddle signals and low-pass filtered at $0.3 \mathrm{~Hz}$. Dashed lines represent the beach toe $(x=8.33 \mathrm{~m}$, and SWL $(x=18.33 \mathrm{~m})$. 


\section{Acknowledgements}

The authors would like to acknowledge the UK Engineering and Physical Sciences Research Council (EPSRC) and HR Wallingford Ltd for their co-sponsorship of the U.K. Coastal Research Facility (UKCRF). The UKCRF tests were undertaken jointly between the Universities of Manchester and Oxford, supported by EPSRC grants GR/N21741 and GR/N22595. The authors would also like to acknowledge Tong Feng and Peter Stansby of the University of Manchester for their part in designing and carrying out the experiments.

\section{References}

Antuono, M., Liapidevskii, V., and Brocchini, M. (2009). Dispersive Nonlinear Shallow-Water Equations. Studies in Applied Mathematics, 122(1), 1-28.

URL https://onlinelibrary.wiley.com/doi/abs/10.1111/j.1467-9590.2008.00422.x

Baldock, T. E. (2006). Long wave generation by the shoaling and breaking of transient wave groups on a beach. Proceedings of the Royal Society A: Mathematical, Physical and Engineering Sciences, 462(2070), $1853-1876$.

Borthwick, A. G. L., and Foote, Y. L. M. (2002). Wave-induced nearshore currents at a tri-cuspate beach in the UKCRF. Proceedings of the Institution of Civil Engineers: Water and Maritime Engineering, 154(4), 251-263.

Borthwick, A. G. L., Ford, M., Weston, B. P., Taylor, P. H., and Stansby, P. K. (2006). Solitary wave transformation, breaking and run-up at a beach. Proceedings of the Institution of Civil Engineers: Maritime Engineering, 159(MA3), 97-105.

Brocchini, M. (2013). A reasoned overview on Boussinesq-type models: the interplay between physics, mathematics and numerics. Proceedings of the Royal Society of London A: Mathematical, Physical and Engineering Sciences, $469(2160)$.

URL http://rspa.royalsocietypublishing.org/content/469/2160/20130496

Bruce, T., Pearson, J., and Allsop, N. W. H. (2002). Hazards at coast and harbour seawalls - velocities and trajectories of violent overtopping jets. Proc. ICCE, ASCE, (pp. 2216-2226).

Da Silva Lima, S. (1981). Wave-induced nearshore currents. PhD Thesis, Liverpool University.

Dalrymple, R. A., and Rogers, B. D. (2006). Numerical modeling of water waves with the SPH method. Coastal Engineering, 53(2), 141-147.

Dalzell, J. F. (1999). A note on finite depth second-order wave-wave interactions. Applied Ocean Research, $21(3), 105-111$.

De Rouck, J., Verhaeghe, H., and Geeraerts, J. (2009). Crest level assessment of coastal structures - General overview. Coastal Engineering, 56(2), 99-107.

Dean, R. G., and Dalrymple, R. A. (1991). Water Wave Mechanics for Engineers and Scientists, vol. Advanced Series on Ocean Engineering, Volume 2. World Scientific.

Dean, R. G., and Sharma, J. N. (1981). Simulation of wave systems due to nonlinear directional spectra. In Proc. Int. Symp. on Hydrodyn. in Oc. Engrg., Norwegian Hydrodynamics Laboratory., (pp. 1211-1222).

Dodd, N. (1998). Numerical model of wave run-up, overtopping, and regeneration. Journal of Waterway, Port, Coastal, and Ocean Engineering, 124(2), 73-81.

Fitzgerald, C. J., Taylor, P. H., Orszaghova, J., Borthwick, A. G. L., Whittaker, C., and Raby, A. C. (2016). Irregular wave runup statistics on plane beaches: Application of a Boussinesq-type model incorporating a generating-absorbing sponge layer and second-order wave generation. Coastal Engineering, 114, 309-324.

Fraccarollo, L., and Toro, E. F. (1995). Experimental and numerical assessment of the shallow water model for two-dimensional dam-break type problems. Journal of Hydraulic Research, 33(6), 843-864. 
Higuera, P., Lara, J. L., and Losada, I. J. (2014). Three-dimensional interaction of waves and porous coastal structures using OpenFOAMß. Part II: Application. Coastal Engineering, 83(Supplement C), 259 - 270. URL http://www.sciencedirect.com/science/article/pii/S0378383913001464

Holman, R. A. (1986). Extreme value statistics for wave run-up on a natural beach. Coastal Engineering, $9(6), 527-544$.

URL http://www.sciencedirect.com/science/article/pii/0378383986900025

Hubbard, M. E., and Dodd, N. (2002). A 2D numerical model of wave run-up and overtopping. Coastal Engineering, 47(1), 1 - 26.

URL http://www.sciencedirect.com/science/article/pii/S0378383902000947

Hughes, S. A. (1993). Physical Models and Laboratory Techniques in Coastal Engineering, vol. 7. World Scientific.

Hunt, A. (2003). Extreme Waves, Overtopping and Flooding at Sea Defences. DPhil Thesis, University of Oxford.

Hunt, I. A. (1959). Design of sea-walls and breakwaters. Transactions of the American Society of Civil Engineers, $126(4), 542-570$.

Hunt-Raby, A. C., Borthwick, A. G. L., Stansby, P. K., and Taylor, P. H. (2011). Experimental measurement of focused wave group and solitary wave overtopping. Journal of Hydraulic Research, 49(4), 450-464.

Jonathan, P., and Taylor, P. H. (1997). On irregular, nonlinear waves in a spread sea. Transactions-American Society of Mechanical Engineers: Journal of Offshore Mechanics and Arctic Engineering, 119, 37-41.

Judge, F., Orszaghova, J., Taylor, P., and Borthwick, A. (2018). A 2DH hybrid Boussinesq-NSWE solver for near-shore hydrodynamics. Coastal Engineering, 142, 9-26.

Judge, F. M. (2018). Two-dimensional horizontal (2DH) Boussinesq modelling of waves at the coast.

Kirby, J. T. (2003). Chapter 1 Boussinesq models and applications to nearshore wave propagation, surf zone processes and wave-induced currents. In V. Lakhan (Ed.) Advances in Coastal Modeling, vol. 67 of Elsevier Oceanography Series, (pp. 1 - 41). Elsevier.

URL http://www.sciencedirect.com/science/article/pii/S0422989403801186

Lara, J. L., Ruju, A., and Losada, I. J. (2010). Reynolds averaged Navier-Stokes modelling of long waves induced by a transient wave group on a beach. Proceedings of the Royal Society A: Mathematical, Physical and Engineering Sciences, 467(2129), 1215-1242.

Liang, Q., and Borthwick, A. G. L. (2009). Adaptive quadtree simulation of shallow flows with wet-dry fronts over complex topography. Computers and Fluids, 38, 221-234.

Lin, P., and Liu, P. L.-F. (1998). A numerical study of breaking waves in the surf zone. Journal of Fluid Mechanics, 359(1), 239-264.

Madsen, P. A., and Schäffer, H. A. (1999). A review of Boussinesq-type equations for surface gravity waves. Advances in Coastal and Ocean Engineering, 5, 1-94.

Madsen, P. A., and Sørensen, O. R. (1992). A new form of the Boussinesq equations with improved linear dispersion characteristics. Part 2. A slowly-varying bathymetry. Coastal Engineering, 18, 183-204.

McAllister, M. L., Adcock, T. A. A., Taylor, P. H., and van den Bremer, T S (2018). The set-down and set-up of directionally spread and crossing surface gravity wave groups. Journal of Fluid Mechanics, 835, $131-169$.

McCabe, M. V., Stansby, P. K., and Apsley, D. D. (2013). Random wave runup and overtopping a steep sea wall: Shallow-water and Boussinesq modelling with generalised breaking and wall impact algorithms validated against laboratory and field measurements. Coastal Engineering, 7/4, 33-49.

Mei, C. C., Stiassnie, M., and Yue, D. K.-P. (1981). Theory and Applications of Ocean Surface Waves: Part 1: Linear Aspects Part 2: Nonlinear Aspects. World Scientific.

Orszaghova, J. (2011). Solitary waves and wave groups at the shore. DPhil Thesis, University of Oxford. 
Orszaghova, J., Borthwick, A. G. L., and Taylor, P. H. (2012). From the paddle to the beach - A Boussinesq shallow water numerical wave tank based on Madsen and Sørensen's equations. Journal of Computational Physics, 231(2), 328-344.

Orszaghova, J., Taylor, P. H., Borthwick, A. G. L., and Raby, A. C. (2014). Importance of second-order wave generation for focused wave group run-up and overtopping. Coastal Engineering, 94(Supplement C), $63-79$.

URL http://www.sciencedirect.com/science/article/pii/S0378383914001598

Panizzo, A., Bellotti, G., and De Girolamo, P. (2002). Application of wavelet transform analysis to landslide generated waves. Coastal Engineering, $44(4), 321-338$.

URL http://www.sciencedirect.com/science/article/pii/S0378383901000400

Rogers, B. D., Fujihara, M., and Borthwick, A. G. L. (2001). Adaptive Q-tree Godunov-type scheme for shallow water equations. International Journal for Numerical Methods in Fluids, 35(3), 247-280.

Schäffer, H. A. (1993a). Infragravity waves induced by short-wave groups. Journal of Fluid Mechanics, 247, $551-588$.

Schäffer, H. A. (1993b). Second Order Irregular-Wave Generation in Flumes-Computation of Transfer Functions by an Asymptotic Summation Method. In Ocean Wave Measurement and Analysis, (pp. 784-797). ASCE.

Schäffer, H. A. (1996). Second-order wavemaker theory for irregular waves. Ocean Engineering, 23(1), 47-88. URL http://www.sciencedirect.com/science/article/pii/002980189500013B

Schäffer, H. A., Madsen, P. A., and Deigaard, R. (1993). A Boussinesq model for waves breaking in shallow water. Coastal Engineering, 20(3-4), 185-202.

Schäffer, H. A., and Steenberg, C. M. (2003). Second-order wavemaker theory for multidirectional waves. Ocean Engineering, 30(10), 1203 - 1231.

URL http://www.sciencedirect.com/science/article/pii/S0029801802001002

Shi, F., Kirby, J. T., Harris, J. C., Geiman, J. D., and Grilli, S. T. (2012). A high-order adaptive timestepping TVD solver for Boussinesq modeling of breaking waves and coastal inundation. Ocean Modelling, $43,36-51$.

Symonds, G., Huntley, D. A., and Bowen, A. J. (1982). Two-dimensional surf beat: Long wave generation by a time-varying breakpoint. Journal of Geophysical Research: Oceans, 87(C1), 492-498.

Tatlock, B., Briganti, R., Musumeci, R. E., and Brocchini, M. (2018). An assessment of the roller approach for wave breaking in a hybrid finite-volume finite-difference boussinesq-type model for the surf-zone. Applied Ocean Research, 73, $160-178$.

URL http://www.sciencedirect.com/science/article/pii/S0141118717306041

Taylor, P. H., and Williams, B. A. (2004). Wave statistics for intermediate depth water - NewWaves and symmetry. Transactions-American Society of Mechanical Engineers: Journal of Offshore Mechanics and Arctic Engineering, 126(1), 54-59.

Tonelli, M., and Petti, M. (2009). Hybrid finite volume - finite difference scheme for 2DH improved Boussinesq equations. Coastal Engineering, 56(5), 609-620.

Tonelli, M., and Petti, M. (2012). Shock-capturing Boussinesq model for irregular wave propagation. Coastal Engineering, 61, 8-19.

Tromans, P. S., Anaturk, A. R., and Hagemeijer, P. (1991). A new model for the kinematics of large ocean waves-application as a design wave. In The First International Offshore and Polar Engineering Conference. International Society of Offshore and Polar Engineers.

van der Meer, J. W., Allsop, N. W. H., Bruce, T., De Rouck, J., Kortenhaus, A., Pullen, T., Schüttrumpf, H., Troch, P., and Zanuttigh, B. (2016). EurOtop: Manual on wave overtopping of sea defences and related structures - An overtopping manual largely based on European research, but for worldwide application.

van der Meer, J. W., and Stam, C. J. M. (1992). Wave runup on smooth and rock slopes of coastal structures. Journal of Waterway, Port, Coastal, and Ocean Engineering, 118(5), 534-550. 
Whittaker, C. N., Fitzgerald, C. J., Raby, A. C., Taylor, P. H., Orszaghova, J., and Borthwick, A. G. L. (2017). Optimisation of focused wave group runup on a plane beach. Coastal Engineering, 121, 44- 55 . URL http://www.sciencedirect.com/science/article/pii/S0378383916304161

Whittaker, C. N., Raby, A. C., Fitzgerald, C. J., and Taylor, P. H. (2016). The average shape of large waves in the coastal zone. Coastal Engineering, 114(Supplement C), $253-264$.

URL http://www.sciencedirect.com/science/article/pii/S0378383916300448

Zhou, Z., Sangermano, J., Hsu, T.-J., and Ting, F. C. K. (2014). A numerical investigation of wave-breakinginduced turbulent coherent structure under a solitary wave. Journal of Geophysical Research: Oceans, $119(10), 6952-6973$. 